\title{
Whitening SOA Testing via Event Exposure
}

\author{
Chunyang Ye and Hans-Arno Jacobsen, Senior Member, IEEE
}

\begin{abstract}
Whitening the testing of service-oriented applications can provide service consumers confidence on how well an application has been tested. However, to protect business interests of service providers and to prevent information leakage, the implementation details of services are usually invisible to service consumers. This makes it challenging to determine the test coverage of a service composition as a whole and design test cases effectively. To address this problem, we propose an approach to whiten the testing of service compositions based on events exposed by services. By deriving event interfaces to explore only necessary test coverage information from service implementations, our approach allows service consumers to determine test coverage based on selected events exposed by services at runtime without releasing the service implementation details. We also develop an approach to design test cases effectively based on event interfaces concerning both effectiveness and information leakage. The experimental results show that our approach outperforms existing testing approaches for service compositions with up to 49 percent more test coverage and an up to 24 percent higher fault-detection rate. Moreover, our solution can trade off effectiveness, efficiency, and information leakage for test case generation.
\end{abstract}

Index Terms-Web service composition, white-box testing, event interface, events

\section{INTRODUCTION}

$\mathrm{T}_{\mathrm{w}}^{\mathrm{H}}$ HE service-oriented architecture (SOA) paradigm is a widely adopted set of software engineering principles to help cope with the complexity of software development for distributed enterprise applications [17], [33]. In this paradigm, service providers develop reusable software components, publish them as Web services, and register them in service registries. By composing selected third-party services from registries, service consumers develop composite SOA applications across distributed, heterogeneous and autonomous organizations [31], [43], [79]. In this paper, unless specifically stated, the term "service consumers" refers to service-oriented application developers who compose third-party services to develop composite SOA applications.

To assure the quality of an SOA application, service consumers usually conduct integration testing to verify whether all the involved services work correctly when composed in the SOA application. Traditional integration testing for component-based systems usually treats components as black-box entities and focuses on the interactions at their interfaces, assuming that all the involved components have been adequately tested before they are integrated [13]. This assumption, however, may not always hold in an SOA context [17]. This is due to the autonomy of service providers and due to the lack of trust between service providers and consumers. Moreover, the SOA paradigm does not offer control to testers or integrators. The lack of control makes it difficult to effectively test SOA applications. Therefore, service consumers have limited insight about whether the integrated third-party services have been

- The authors are with the Department of Electrical and Computer Engineering, University of Toronto, Canada.

E-mail: chunyang@msrg.utoronto.ca, jacobsen@eecg.toronto.edu.

Manuscript received 8 Jan. 2012; revised 30 Sept. 2012; accepted 1 Apr. 2013; published online 5 Apr. 2013

Recommended for acceptance by E. Di Nitto.

For information on obtaining reprints of this article, please send e-mail to: tse@computer.org, and reference IEEECS Log Number TSE-2012-01-0009.

Digital Object Identifier no. 10.1109/TSE.2013.20. adequately tested and therefore are unable to determine how well the SOA application as a whole has been tested [4].

Whitening SOA testing by examining test coverage of the internal program structure of an SOA application could provide service consumers with confidence on how adequately the SOA application has been tested. Measuring test coverage based on program structure such as data-flow or control-flow is a widely adopted technique to quantify how well a program has been tested [13], [83]. However, measuring test coverage of an SOA application based on its internal program structure is a difficult task when the SOA application integrates third-party services from different providers.

To protect their intellectual property base, service providers seldom reveal the implementation of a service to their consumers. This prevents service consumers from understanding the program structure of third-party services and measuring their coverage during testing.

To address this dilemma, Bartolini et al. [4] suggested that each third-party service provider report coverage information for its services to service consumers for testing purposes, such as the percentage of code paths covered, instead of revealing the service's implementation. Based on the coverage information reported and the given coverage criteria, service consumers can estimate how well each service has been tested at the unit testing level.

Although whitening the unit testing of individual thirdparty services provides service consumers confidence on how well each third-party service has been tested, we observe that reporting only the coverage percentage of each third-party service is not enough for integration testing of a service composition involving more than one third-party service. There are two main reasons for this: 1 ) the inability to accurately determine test coverage as a whole and 2) the difficulty to effectively design test cases.

First, although service consumers can obtain the coverage percentage of every third-party service involved in an SOA application, service consumers are still unable to 
accurately determine how well the SOA application as a whole has been tested. Let us take the path coverage criterion as an example [83]. Given $m$ services, each of which has $n_{i}$ paths, ${ }^{1}$ their composition may have up to $\prod_{1}^{m} n_{i}$ paths [13]. According to the anticomposition axiom [72], even if the paths of each service are well covered in testing, the service composition as a whole may not be adequately tested. As a result, faults in untested execution scenarios in the service composition are left undetected (for an illustration of this, refer to Section 2).

Second, it is difficult for service consumers to effectively design test cases to cover a service composition. For example, with respect to path coverage [83], since the execution of a path in one service may depend on the execution of a particular path in another service [13], [72], it is impossible to derive this dependence information from the coverage information reported by service providers alone. As a result, service consumers may need to try a large number of test cases to cover all possible paths, requiring a significantly increased testing effort.

Therefore, reporting only the coverage percentage of each third-party service for integration testing is not enough. This observation prompted us to explore what other information services could reveal to whiten the integration testing of the compositions they partake in while keeping their implementation details hidden from service consumers.

We observe that at runtime the execution of a service may generate many events to reflect the state changes inside the service [21], [47] (e.g., the Apache ODE BPEL engine [1] generates events once the value of a variable changes or a task is initiated or completed, etc.). The events generated at runtime are informative because they provide a partial view of a service's runtime behavior. Therefore, in this paper we explore the potential of allowing services to expose events to whiten the testing of service compositions.

In our approach, instead of reporting the coverage percentage, service providers select from events generated at runtime and expose them to service consumers via event interfaces, an abstraction we introduce later on more formally. The event interface of a service advertises what kinds of events the service exposes at runtime as well as the relationship between the exposed events. In this way, service consumers can make use of the exposed events and the event interfaces to reason about how well a service composition has been covered in testing and derive test cases effectively to test uncovered scenarios.

The main challenges of this approach lie in how to trade off the benefits of event exposure for testing and the side effects such as information leakage about a service's implementation (e.g., backend states and workflow, etc.). In particular, we pursue the following three questions: 1) How do we encapsulate and expose only the necessary events from a large number of events generated by services at runtime to hide service implementation details; 2) how do we correlate events from different services to reason about the test coverage of a service composition as a whole; and 3) how do we make use of the exposed events to design test cases to effectively cover a service composition.

1. The issue of handing an infinite number of paths for the path coverage is discussed in Section 6.1.2.
On the one hand, exposing too many events may provide enough information for service consumers to accurately determine the test coverage of a service composition and design test cases effectively, but the overexposed events may also allow service consumers to understand how a third-party service is implemented and thus cause information leakage for service providers. On the other hand, exposing fewer events may provide too limited information for service consumers to reason about the test coverage and to effectively design test cases. Therefore, how to encapsulate and expose only necessary events for testing purposes while protecting service implementation details from being leaked is a challenging question.

We address these challenges in this paper with a fivefold contribution: First, we propose a novel approach to whiten the testing of SOA applications involving more than one third-party service via events exposed from services. This approach allows service consumers to gain confidence on how well an SOA application has been tested. Second, we develop a model to derive event interfaces from service implementations to expose only necessary events. We illustrate that the test coverage calculated based on exposed events can be used to reason about the real test coverage of an SOA application without revealing the service implementation details. Third, we devise an approach to hide information from exposed events based on event encapsulation to reduce information leakage. Fourth, we propose approaches to derive test cases based on event interfaces concerning both the test effectiveness and information leakage. This allows service providers to customize their services with different testing capacity requirements. Finally, we perform a detailed experimental evaluation resulting in the following key insights. The experimental results show that our approach can achieve up to 49 percent more test coverage and detect up to 24 percent more faults than existing approaches. The results also show that our solution can trade off effectiveness, efficiency, and information leakage in test case generation.

The remainder of this paper is organized as follows: Section 2 motivates the research problem using an example. Section 3 outlines our solution and research questions. Section 4 formalizes the notions of Web service and event underlying the analysis in this paper. Section 5 introduces two event-based coverage criteria. Section 6 presents our approach to expose events and reason about the test coverage based on exposed events. Section 7 demonstrates how to trade off the accuracy of coverage reasoning and information leakage. Section 8 introduces our solution to design test cases effectively. Section 9 evaluates our approach empirically and Section 10 discusses some limitations. Finally, Section 11 positions our work in the broader context of related work.

\section{Motivating Example}

As discussed in Section 1, testing an SOA application comprised of third-party services is a paradox: On the one hand, white-box testing an SOA application requires the implementation details of every third-party service involved, which, to protect the intellectual property base of a service provider, are kept hidden from a service consumer. 


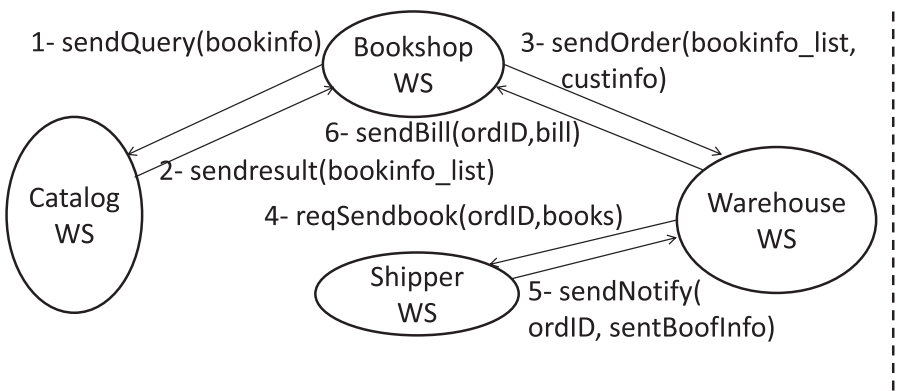

(a) Collaboration diagram

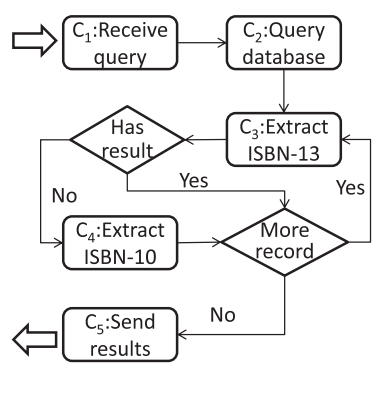

(b) Catalog WS workflow

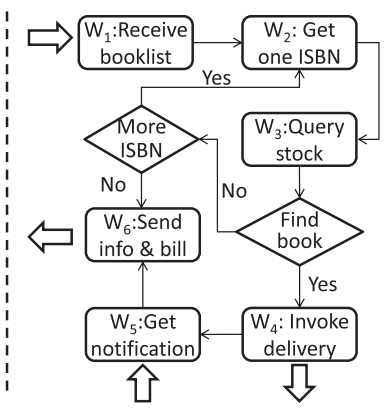

(c) Warehouse WS workflow

Fig. 1. Service composition example: online bookshop application.

On the other hand, black-box testing an SOA application requires no implementation details of third-party services to become visible but suffers from the limitation that little assurance can be offered as to the overall test coverage ${ }^{2}$ of the SOA application. As a result, existing approaches to testing SOA applications [4], [17], [46], [55], [56] either opt to expose all involved third-party services or lack assurance about the quality of testing, leaving severe faults undetected.

In this section, we use an online book sales application scenario adapted from [2] to illustrate this dilemma. Fig. 1a shows the composition of four Web services (WS) that implement the application. The Bookshop WS sets out to find the cheapest book matching the customers' search criteria. On receiving an order, the Bookshop WS invokes the Catalog WS to retrieve ISBN information of the ordered book based on book title. Then, the Bookshop WS sorts the returned list of ISBN numbers based on price and places an order to the Warehouse WS with the sorted list. The Warehouse WS queries the books from the sorted list one by one based on ISBN numbers and picks the first book available in stock. Next, the Shipper WS is invoked to deliver the selected book to the customer. Finally, the Warehouse WS forwards an invoice to the customer for payment. In this application, the four Web services belong to different organizations and their implementation details are not exposed. For illustration purposes, we present the workflow of the Catalog WS and the Warehouse WS in Figs. $1 \mathrm{~b}$ and $1 \mathrm{c}$, respectively.

A service composition is incorrect (i.e., fails to perform the correct operation) if a customer receives a book that is different from the one ordered or is not the cheapest one in stock. For example, suppose the ordered book has recently been republished by a publisher and is sold at a discount (i.e., it is cheaper than other versions of the book). The newly published version of the book includes ISBN numbers according to two number formatting standards, say ISBN-13 and ISBN-10, where ISBN-13 is a new standard with a 13 digit number format, while ISBN-10 is the old standard with a number format comprising 10 digits. The Catalog WS queries the database with the title of the book and extracts ISBN-13 for each matching record. If ISBN-13 is not available, then ISBN-10 is used instead. However, the Warehouse WS is based on a legacy system that supports

2. This refers to code-based structural coverage criteria only, not necessarily to specification-based coverage criteria. querying with ISBN-10 only. ${ }^{3}$ As a result, although the cheapest book is in stock, the Warehouse WS cannot find it based on ISBN-13. Instead, the Warehouse WS picks the more expensive book based on ISBN-10 from the list of matching records and delivers it to the customer.

Since this failure ${ }^{4}$ is caused by incompatible data formats used by two autonomous Web services (i.e., one uses ISBN-13 whereas the other one uses ISBN-10), testing the involved Web services in isolation does not suffice to determine the incompatibility problem. Also, existing integration testing approaches for Web service compositions treat Web services as black-box components and verify the correctness of their choreography only [9], [42], [57], [56]. According to the antidecomposition axiom [72], verifying the choreography cannot guarantee that a service composition has been adequately tested. For example, the choreography in Fig. 1a can be adequately covered (in terms of path coverage) in testing if the execution of the Catalog WS and the Warehouse WS follows the paths " $C_{1}, C_{2}, C_{3}, C_{4}, C_{5}$ " and " $W_{1}, W_{2}, W_{3}, W_{4}, W_{5}, W_{6}$," respectively. However, the aforementioned fault in the application still cannot be detected unless both services follow the path " $C_{1}, \ldots, C_{2}, C_{3}, \ldots, C_{5}$ " and " $W_{1}, \ldots, W_{2}, W_{3}, \ldots, W_{4}$, $W_{5}, W_{6}$." Since services are treated as black-box components, service consumers cannot determine the hidden scenarios by examining the service choreography alone, which describes only a partial and abstract view of the entire service composition. As a result, a service composition as a whole may not be adequately tested to reveal residual faults.

In addition, even though the aforementioned hidden scenario is known, an execution of the service composition that covers this scenario may depend on other factors (e.g., database of Catalog WS, stock in Warehouse WS and business logic of Bookshop WS, etc.) which are invisible to service consumers. As a result, service consumers may still need to try a large number of test cases to cover the given scenario, significantly increasing the testing effort.

In the next section, we summarize our solution and demonstrate how we address the "event exposure versus information leakage" dilemma by selectively exposing events from within services.

3. Note that the inconsistency in querying with ISBN-10 and ISBN-13 is not uncommon, and a report of such inconsistency by practitioners can be found at https://jira.sakaiproject.org/browse/UMICH-25.

4. The test oracle issue is out of the scope of this paper. With respect to this example, the tester can verify the testing result with the list of available books provided by the Warehouse WS. We present more discussion about the test oracle issue in Section 10. 

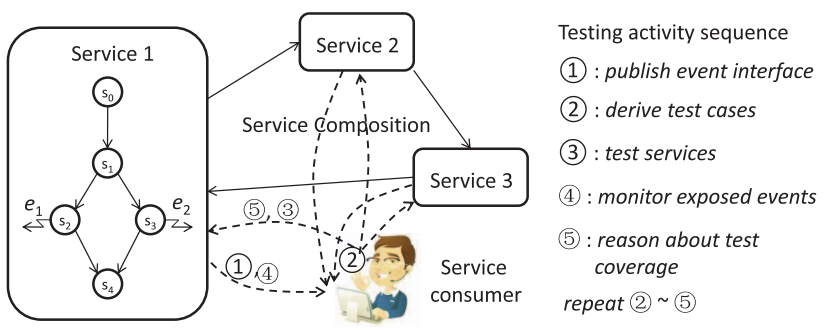

Fig. 2. Solution overview.

\section{Overview of Methodology}

In this section, we give an overview of our methodology from the perspective of service consumers and providers, respectively. We first demonstrate how our approach can be used by service consumers to test SOA applications. Then, we review the basic ideas behind our solution and discuss the research questions addressed in the remainder of this paper.

\subsection{Usage Illustration}

Fig. 2 illustrates how service consumers apply our approach to test SOA applications:

1. The service provider of each involved third-party service exposes an event interface to service consumers. The event interface declares what kinds of events the service provider exposes at runtime.

2. Service consumers collect all the event interfaces from third-party service providers and derive test cases to test the SOA application.

3. Service consumers test the SOA application using the derived test cases.

4. Service consumers subscribe to and monitor the events exposed by all third-party services.

5. Service consumers make use of the events from third-party services to reason about the test coverage of the SOA application.

Steps 2-5 are repeated until a given coverage criterion is satisfied or a given number of rounds have been completed.

Note that a service composition can be tested from two different perspectives, namely, service orchestration and service choreography [6], [16]. A service orchestration represents a service composition from one involved service's perspective, describing how this involved service is constructed and how it interacts with other services involved in the service composition [64]. Instead of viewing a service composition from any particular involved service's perspective, a service choreography describes how all the involved services collaborate and interact with each other from a global perspective [64].

Our approach can be applied to both service orchestration and service choreography. With respect to a service orchestration, service consumers are the developers or testers of the particular service orchestration. With respect to a service choreography, service consumers can be a thirdparty tester or all the involved service providers (e.g., they collaborate to test the service composition together). In either case, service consumers can apply our approach to test a service composition following the same steps as illustrated in Fig. 2.

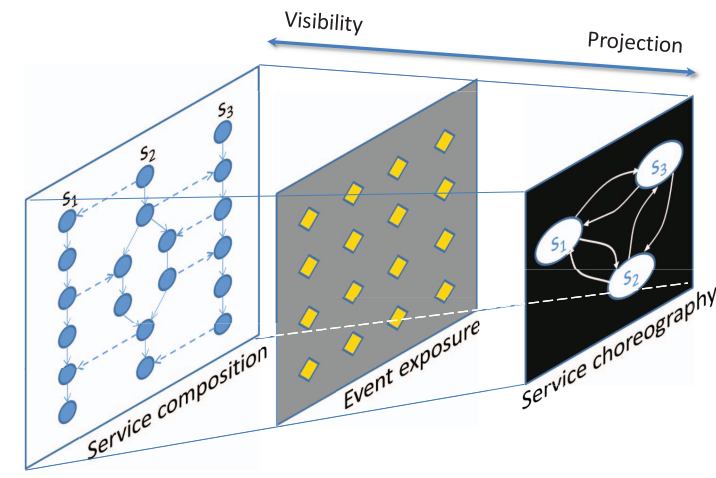

Fig. 3. Illustration of event exposure at a conceptual level.

In addition, a third-party service may be discovered dynamically and bound to a service composition at runtime. To apply our approach to such a scenario, the third-party service needs to register its event interface in the public registries (e.g., tModel in UDDI) and explicitly specify available invocation modes (e.g., testing modes [18]) in the service registries.

\subsection{Solution Overview and Research Questions}

Here, we summarize the idea of exploring the use of exposing events from within services to whiten the testing of service compositions. All notions informally introduced below are formalized in Section 4. The term "event" used in this paper is defined as a state change [21], [47]. A state of a service is defined as a snapshot of its execution at runtime. The execution of a service can be seen as a series of transitions among its states. The transition from one state to another is defined as a state change. For example, an online shopping service transitions from the state "the customer has not been verified" to "the customer has been verified." Usually, these states are invisible from outside the service and thus are referred to as internal states. We define an event to reveal or to expose a state change from within a service.

Our basic idea is to abstract coverage-related internal state changes as events and expose them to service consumers to increase the visibility in testing. As illustrated in Fig. 3, the entire backend state space of a service composition is invisible to service consumers except for the service choreography among the involved services, which is an abstract and partial view of the service composition. In our approach, we suggest service providers expose some events generated at runtime to service consumers so that service consumers can make use of the exposed events to gain more visibility and assurance on how adequately the service composition has been tested. Service providers can choose to expose the events that can be used to indicate the test coverage in testing.

For example, if the Catalog WS exposes an event indicating that the execution of an internal $\operatorname{task}^{5} C_{3}$ is completed, then service consumers can identify that task $C_{3}$ is covered in testing when observing this event. Similarly, if

5. In a Web service composition, Web services communicate with each other through port actions that are defined in their WSDL interfaces [68]. Tasks other than port actions inside a Web service do not participate in any communications with other services. These tasks are invisible to service consumers and are referred to as internal tasks. 
the Warehouse WS exposes two events representing the completion of internal tasks $W_{2}$ and $W_{3}$, respectively, service consumers can derive whether a path like " $W_{1}, \ldots, W_{2}$, $W_{3}, \ldots$ " is covered or not. In addition, service consumers can also track data flow covering if service providers expose a pair of events (e.g., $e_{\text {def }}, e_{u s e}$ ) for the execution of every two tasks that define and use a variable of interest in the service composition (i.e., define-use relations [83]). In Section 6, we illustrate how to make use of events to represent different test coverage criteria [83].

The main challenge of our approach lies in how to balance the tradeoff between the visibility needed to increase test coverage and the lack of visibility required by service providers who do not wish to reveal service internals. As illustrated in Fig. 3, if service providers expose a small number of events to service consumers, service consumers may only gain limited visibility improvements for testing. On the other hand, exposing too many events may provide enough visibility for service consumers to accurately determine test coverage for a service composition, but the overexposure of events may also allow service consumers to understand how a third-party service is implemented. This is the main challenge addressed in the remainder of this paper. In particular, we strive to answer the following three research questions in this paper:

Q1: How do we encapsulate and expose events? A service provider needs to expose only necessary events to reveal test coverage information and to encapsulate exposed events to suppress irrelevant and proprietary service details. We propose an approach to address this issue in Sections 6 and 7.

Q2: How do we reason about test coverage of a service composition based on exposed events? Service consumers need to derive how adequately an SOA application has been tested based on events observed during testing. We show how to do so in Sections 5 and 6.

Q3: How do we make use of exposed events to design test cases to effectively cover a service composition? Besides event exposure and determination of test coverage, an approach is also needed to design test cases to cover a service composition effectively, which we undertake in Section 8.

\section{Web Services and Events}

Before illustrating how to whiten the testing of service compositions based on events exposed by services, we define some basic notions. Also, to keep the paper selfcontained, this section reviews and formalizes the concepts used in the remainder of this paper, including Web service, service composition, and event. For illustration purposes, similar to many existing approaches [12], [32], [33], [79], we model a service as a finite state machine. Our solution, however, is not restricted to this model. In Section 10, we discuss how to apply our approach to other programming models (e.g., BPEL and Java).

Definition 1 (State). A state $s$ is defined as a finite set $\left\{\left(x_{1}, t p_{1}, v_{1}\right), \ldots,\left(x_{n}, t p_{n}, v_{n}\right)\right\}(n>0)$, where $x_{i}$ is a variable, and $t p_{i}$ and $v_{i}$ are its type and value. ${ }^{6}$

6. Note that the value of a variable can be a concrete value or a constraint to define a set of values (e.g., $v_{i} \equiv x_{i}>0$ ).

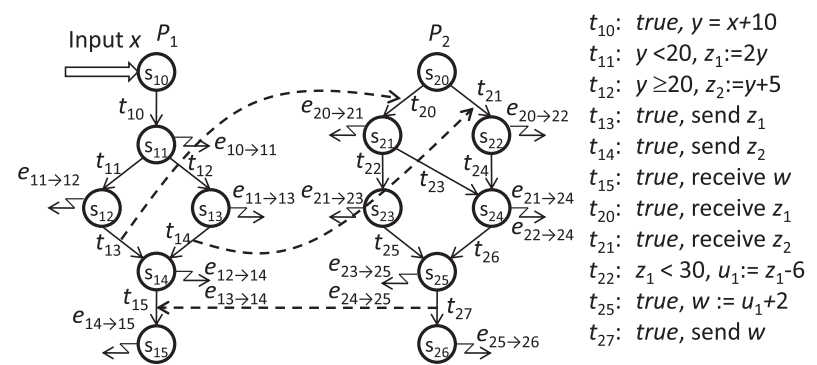

Fig. 4. Web service composition and event exposure.

Definition 2 (Service). A service $P$ is a six tuple $\left(S, s_{0}, G\right.$, $C \cup I, T, F)$, where $S$ is a set of states, $s_{0} \in S$ is the initial state, $F \subseteq S$ is the set of final states, $C$ is the set of port actions ${ }^{7}$ (e.g., sending or receiving a message), $I$ is the set of internal actions invisible to service consumers, $G$ is the set of guarded Boolean expressions, and $T \equiv S \times G \times(C \cup I) \times S$ represents the set of transitions.

Given a state $s$ in service $P, P$ can transition from $s$ to $s^{\prime}$, denoted as $s \stackrel{t}{\rightarrow} s^{\prime}$, if and only if $\exists t \equiv\left(s, g, a, s^{\prime}\right) \in T \wedge$ $s \vdash g$. An execution of $P$ (also denoted as an instance of $P$ ) is a sequence of $s_{0} \stackrel{t_{i 1}}{\rightarrow} s_{i 1} \stackrel{t_{i 2}}{\rightarrow} \cdots \stackrel{t_{i k}}{\rightarrow} s_{i k}$, where $s_{i k}$ is its current state. The execution of a service is the transitioning of the service from one state to another. Informally speaking, these state changes represent that something happened and are defined as events.

Definition 3 (Event). Let $s \equiv\left\{\left(x_{1}, t p_{1}, v_{1}\right), \ldots,\left(x_{n}, t p_{n}, v_{n}\right)\right\}$ and $s^{\prime} \equiv\left\{\left(x_{1}^{\prime}, t p_{1}^{\prime}, v_{1}^{\prime}\right), \ldots,\left(x_{n}^{\prime}, t p_{n}^{\prime}, v_{n}^{\prime}\right)\right\}$ be two states of service $P$. A state change from $s$ to $s^{\prime}$ is defined as an event $e_{s \rightarrow s^{\prime}} \equiv\left\{\left(x_{i 1}, t p_{i 1}, v_{i 1}\right), \ldots,\left(x_{i k}, t p_{i k}, v_{i k}\right)\right\} \subseteq s \cup s^{\prime}$.

For example, as illustrated in Fig. 4, we can define an event $e_{10 \rightarrow 11}$ to represent the state change from $s_{10}$ to $s_{11}$ in service $P_{1}$. Note that an event is different from a transition, in the sense that the former defines that something of interest happens (i.e., a state change related to a set of variables $x_{i 1}, \ldots, x_{i k}$ of interest), whereas the latter defines how something happens (i.e., how a state change comes about). Since our purpose is to determine the coverage of a service composition in testing without revealing the implementation details of services, we only need to know the state changes related to coverage in testing. Therefore, we suggest encapsulating events to filter irrelevant state details and exposing them to indicate the test coverage inside a service (illustrated in Section 6).

Given a set of Web services, their composition results from wiring the port actions between the services (e.g., the dashed lines in Fig. 4 between $P_{1}$ and $P_{2}$ ). The link between two port actions from two different services represents a message exchange between the two services, as specified in their WSDL interfaces [68]. To ease the presentation, we denote the port action sending message $m_{h}$ (the index $h$ represents a message type) from service $P_{i}$ to service $P_{j}$ as $! m_{h, i \rightarrow j}$ and the port action from service $P_{j}$ receiving message $m_{h}$ as $? m_{h, i \rightarrow j}$. The following definition specifies the behavior of a service composition.

7. In this paper, the term "action" is used interchangeably with the terms "activity" in BPEL and "task" in traditional workflows. 
Definition 4 (Service Composition). Given $n$ services $P_{i} \equiv$ $\left(S_{i}, s_{i, 0}, G_{i}, C_{i} \cup I_{i}, T_{i}, F_{i}\right), \quad i=1 . . n$, their composition is denoted as a service $P \equiv \oplus\left(P_{1}, P_{2}, \ldots, P_{n}\right)$. A state of $P$ can be represented as $\left(\left(s_{1, c_{1}}, Q_{1}\right), \ldots,\left(s_{n, c_{n}}, Q_{n}\right)\right)$, where $s_{i, c_{i}} \in S_{i}$, and $Q_{i}$ is the queue of incoming messages from partner services. The transition $\left(\left(s_{1, c_{1}}, Q_{1}\right), \ldots,\left(s_{n, c_{n}}, Q_{n}\right)\right) \rightarrow\left(\left(s_{1, c_{1}}^{\prime}, Q_{1}^{\prime}\right)\right.$, $\left.\ldots,\left(s_{n, c_{n}}^{\prime}, Q_{n}^{\prime}\right)\right)$ is allowed if and only if any of the following conditions are satisfied:
1. $\exists t_{i} \equiv\left(s_{i, c_{i}}, g_{i}, a_{i}, s_{i, c_{i}}^{\prime}\right): s_{i, c_{i}} \stackrel{t_{i}}{\rightarrow} s_{i, c_{i}}^{\prime} \wedge a_{i} \in I_{i} \wedge(\forall l \neq$ $\left.i: s_{l, c_{l}}^{\prime}=s_{l, c_{l}}\right) \wedge\left(\forall l: Q_{l}^{\prime}=Q_{l}\right)$.
2. $\exists t_{i} \equiv\left(s_{i, c_{i}}, g_{i}, a_{i}, s_{i, c_{i}}^{\prime}\right): s_{i, c_{i}} \stackrel{t_{i}}{\rightarrow} s_{i, c_{i}}^{\prime} \wedge a_{i}=! m_{h, i \rightarrow j} \wedge$ $Q_{j}^{\prime}=Q_{j} m_{h, i \rightarrow j} \wedge\left(\forall l \neq i: s_{l, c_{l}}^{\prime}=s_{l, c_{l}}\right) \wedge(\forall l \neq j:$ $\left.Q_{l}^{\prime}=Q_{l}\right)$.
3. $\exists t_{j} \equiv\left(s_{j, c_{j}}, g_{j}, a_{j}, s_{j, c_{j}}^{\prime}\right): s_{j, c_{j}} \stackrel{t_{j}}{\rightarrow} s_{j, c_{j}}^{\prime} \wedge a_{j}=? m_{h, i \rightarrow j} \wedge$ $m_{h, i \rightarrow j} Q_{j}^{\prime}=Q_{j} \wedge\left(\forall l \neq j: s_{l, c_{l}}^{\prime}=s_{l, c_{l}} \wedge Q_{l}^{\prime}=Q_{l}\right)$.

Intuitively, Definition 4 specifies how a service composition as a whole transitions from one state to another. In particular, Condition 1 represents a transition by executing an internal action (that is, from $I_{i}$ ) of an involved service $P_{i}$; Condition 2 represents a transition that service $P_{i}$ sends a message $m_{h}$ to service $P_{j}$ (the message $m_{h}$ is attached to the tail of $Q_{j}$ ); Condition 3 represents a transition that service $P_{j}$ handles the message $m_{h}$ received from service $P_{i}$ (the message $m_{h}$ is removed from the head of $\left.Q_{j}\right)$.

A state $\left(\left(s_{1, c_{1}}, Q_{1}\right), \ldots,\left(s_{n, c_{n}}, Q_{n}\right)\right)$ is called feasible if and only if there exists a sequence of transitions $\left(\left(s_{1,0},\{\}\right), \ldots\right.$, $\left.\left(s_{n, 0},\{\}\right)\right) \rightarrow \cdots \rightarrow\left(\left(s_{1, c_{1}}, Q_{1}\right), \ldots,\left(s_{n, c_{n}}, Q_{n}\right)\right)$.

\section{Event-Based Coverage Criteria}

Given the events exposed by services, in this section we discuss how to make use of these events to measure test coverage.

Since the services involved in a service composition declare what kinds of events they expose at runtime (also referred to as event advertisement), one simple coverage criterion for a service composition is to examine whether all the events advertised by services are exposed and observed in testing. We call this criterion event coverage.

Event coverage criterion (ECC). Given a service composition $P$ involving services $P_{i}, i=1 . . n$ and let $E D_{i}$ be the set of events advertised by $P_{i}$ (i.e., the set of events that can be exposed by service $\left.P_{i}\right)$. Suppose $E P_{i}\left(\subseteq E D_{i}\right)$ is the set of events exposed by $P_{i}$ and observed by service consumers under a given set of test cases $t c$, the event coverage percentage, denoted as $C_{e c}(P, t c)$, is equal to $\left|\cup_{i=1}^{n} E P_{i}\right| /\left|\cup_{i=1}^{n} E D_{i}\right|$.

For example, suppose services $P_{1}$ and $P_{2}$ in Fig. 4 advertise events $e_{10 \rightarrow 11}, e_{11 \rightarrow 12}, e_{11 \rightarrow 13}$, and $e_{21 \rightarrow 23}, e_{21 \rightarrow 24}$, $e_{22 \rightarrow 24}$, respectively. During testing, if $e_{10 \rightarrow 11}, e_{11 \rightarrow 12}$, and $e_{21 \rightarrow 23}$ are observed, then 50 percent of the advertised events are covered. Intuitively, the exposed events reveal some internal state changes inside a Web service and can thus be used to indicate the test coverage of the service. For instance, $e_{10 \rightarrow 11}$ indicates that the task in $t_{10}$ is covered; $e_{11 \rightarrow 12}$ and $e_{11 \rightarrow 13}$ imply the branch coverage of $t_{11}$ and $t_{12}$, respectively. In more general cases, service providers can advertise and expose events under particular conditions (e.g., an event indicating that a book is out of stock, etc.). Therefore, service consumers can gain visibility and confidence on how adequately a service composition has been tested by measuring how many advertised events are observed in testing.

Examining whether each individual event advertised by the involved services is observed during testing can give service consumers confidence on the adequacy of testing. However, this simple approach may still be insufficient in detecting faults that cut across the involved services (e.g., inconsistency faults as introduced in Section 2) because each individual event represents a local state change inside the involved service. Therefore, we propose another eventbased coverage criterion, namely, event sequence coverage, to measure the coverage of a service composition from a global perspective. With event sequence coverage, service consumers can correlate events advertised by the involved services to enumerate all the possible event sequences they want to observe based on the temporal order of the exposed events. Test adequacy is then evaluated based on how many event sequences can be observed in testing.

We measure the temporal order of events based on their causality relationship. Formally, given two events $e_{s_{1} \rightarrow s_{2}}$ and $e_{s_{3} \rightarrow s_{4}}$ raised in a service composition $P \equiv \oplus\left(P_{1}\right.$, $\left.P_{2}, \ldots, P_{n}\right), e_{s_{1} \rightarrow s_{2}}$ is said to precede $e_{s_{3} \rightarrow s_{4}}$, denoted as $e_{s_{1} \rightarrow s_{2}} \ll e_{s_{3} \rightarrow s_{4}}$, if and only if any of the following conditions is satisfied:

- $\quad e_{s_{1} \rightarrow s_{2}}$ and $e_{s_{3} \rightarrow s_{4}}$ are from the same service $P_{i}$ and $\exists s_{i, 1} \stackrel{t_{1}}{\rightarrow} s_{i, 2} \stackrel{t_{2}}{\rightarrow} \cdots \stackrel{t_{i, k-1}}{\rightarrow} s_{i, k} \wedge s_{i, 1} \equiv s_{2} \wedge s_{i, k} \equiv s_{3}$.

- $\quad e_{s_{1} \rightarrow s_{2}}$ is from service $P_{i}$ where

$$
\begin{aligned}
& \exists s_{i, 1} \stackrel{t_{i, 1}}{\rightarrow} s_{i, 2} \stackrel{t_{i, 2}}{\rightarrow} \cdots \stackrel{t_{i, k}}{\rightarrow} s_{i, k+1} \wedge s_{i, 1} \equiv s_{1} \\
& \quad \wedge s_{i, r} \equiv s_{2}(1<r \leq k+1) \wedge t_{i, k} \\
& \quad=\left\{\left(s_{i, k}, g_{i, k}, a_{i, k}, s_{i, k+1}\right)\right\} \wedge a_{i, k}=! m_{h, i \rightarrow j},
\end{aligned}
$$

and $e_{s_{3} \rightarrow s_{4}}$ is from service $P_{j}(i \neq j)$ where

$$
\begin{aligned}
& \exists s_{j, 1} \stackrel{t_{j, 1}}{\rightarrow} s_{j, 2} \stackrel{t_{j, 2}}{\rightarrow} \cdots \stackrel{t_{j, l}}{\rightarrow} s_{j, l+1} \wedge s_{j, l+1} \\
& \quad \equiv s_{4} \wedge s_{j, o} \equiv s_{3}(1 \leq o<l+1) \wedge t_{j, 1} \\
& \quad=\left\{\left(s_{j, 1}, g_{j, 1}, a_{j, 1}, s_{j, 2}\right)\right\} \wedge a_{j, 1}=? m_{h, i \rightarrow j} .
\end{aligned}
$$

Condition 1 indicates that two events are raised sequentially by a service (e.g., $e_{10 \rightarrow 11} \ll e_{11 \rightarrow 12}$ in Fig. 4). ${ }^{8}$ Condition 2 indicates that $e_{s_{1} \rightarrow s_{2}}$ represents a state change inside service $P_{i}$ before (or right after) $P_{i}$ sends a message to service $P_{j}$, and $e_{s_{3} \rightarrow s_{4}}$ represents a state change inside $P_{j}$ after $P_{j}$ receives a message from $P_{i}$. Therefore, logically $e_{s_{1} \rightarrow s_{2}}$ should occur before $e_{s_{3} \rightarrow s_{4}}$. For instance, in Fig. $4, e_{10 \rightarrow 11} \ll$ $e_{20 \rightarrow 21}$ because $e_{10 \rightarrow 11}$ is raised before $P_{1}$ sends message $z_{1}$, whereas $e_{20 \rightarrow 21}$ is raised after $P_{2}$ receives $z_{1}$.

Based on the temporal order of events, we can organize events into a sequence. An event sequence $e_{1}, e_{2}, \ldots, e_{m}$ is feasible if and only if $\neg \exists i, j:(i<j) \wedge e_{j} \ll e_{i}$. A feasible event sequence defines a feasible order in which the events can be raised by a service composition. Note that since the involved services in a service composition may be executed concurrently, events from different services can be exposed simultaneously. Logically these simultaneously exposed events may not have any causality relationship. As a result, these events can be combined in different orders to form

8. Section 6.2 shows how a service can reveal the temporal order of its events to service consumers via its event interface. 
different feasible event sequences. However, we are not interested in differentiating these combinations and covering all of them in testing because there are no logical dependences among these events. Moreover, service consumers may not be able to identify which of these events are raised first due to the distributed nature of service compositions. Therefore, we propose the following definition to classify these different combinations as identical.

Definition 5 (Identical Event Sequence). Two event sequences $e_{1}, e_{2}, \ldots, e_{m}$ and $e_{1}^{\prime}, e_{2}^{\prime}, \ldots, e_{m}^{\prime}$ are identical if and only if the following conditions are satisfied:

- $\forall e_{i}:\left(\exists e_{j}^{\prime}: e_{i} \equiv e_{j}^{\prime}\right)$.

- $\forall e_{i}^{\prime}:\left(\exists e_{j}: e_{i}^{\prime} \equiv e_{j}\right)$.

- $\forall e_{i} \equiv e_{j}^{\prime}, e_{k} \equiv e_{l}^{\prime}: e_{i} \ll e_{k} \leftrightarrow e_{j}^{\prime} \ll e_{l}^{\prime}$.

Based on the above discussion, we formulate the following criterion to examine how adequately the advertised event sequences are observed in testing.

Event sequence coverage criterion (ESCC). Given a service composition $P$, let $F E S$ be the set of unique feasible event sequences (i.e., no identical event sequences) that service consumers want to observe. Suppose $O E S$ is the set of unique feasible event sequences observed by service consumers under a given set of test cases $t c$ in testing. The event sequence coverage percentage for testing, denoted as $C_{e s c}(P, t c)$, is equal to $|O E S| /|F E S|$.

For example, the service composition in Fig. 4 may advertise the following feasible event sequences: $e_{10 \rightarrow 11}, e_{11 \rightarrow 12}, e_{12 \rightarrow 14}, e_{20 \rightarrow 21}, e_{21 \rightarrow 23}, e_{23 \rightarrow 25}, e_{25 \rightarrow 26}, e_{14 \rightarrow 15}$ and $e_{10 \rightarrow 11}, e_{11 \rightarrow 13}, e_{13 \rightarrow 14}, e_{20 \rightarrow 22}, e_{22 \rightarrow 24}, e_{24 \rightarrow 25}, e_{25 \rightarrow 26}, e_{14 \rightarrow 15}$, each of which indicates an execution path in the service composition. Therefore, ESCC can be used to measure the test adequacy of a service composition. Note that when the event sequence has only one event, ESCC is equal to ECC. Therefore, ECC is a special case of ESCC. In the remainder of this paper, we consider ESCC only.

With the event-based coverage criteria proposed in this section, service consumers can calculate how adequately a test run satisfies the given criterion based on the exposed events. In the extreme case where service providers expose all the events generated during the execution of every statement inside the services, the event-based coverage criteria can be used to measure the actual coverage of program code inside a service composition (e.g., statement coverage, path coverage [83], etc.). However, service providers may not be willing to expose all the events but only a restricted subset of them due to business interests such as the prevention of information leakage. Therefore, we need to answer the following question: Is the measurement using event-based coverage criteria still meaningful when service providers expose only a subset of events? That is, does the test coverage calculated based on events still reflect the real coverage of service implementations inside the service composition? In the next section, we investigate this issue.

\section{Coverage Reasoning}

By observing the events exposed by service providers in testing, service consumers can calculate the test coverage using the event-based coverage criteria proposed in Section 5 .
In this section, we investigate how to make the measurement of test coverage based on events meaningful, in the sense that the coverage calculated based on events reflects the actual test coverage of a service composition.

\subsection{Structure-Based Coverage Criteria}

To measure the actual test adequacy of a service composition as a whole, we adopt the structure-based coverage criteria from traditional software testing. In particular, without loss of generality, we consider two representative structure-based data flow and control flow coverage criteria: They are representative of cases where one should track the definitions and uses of variables and cases where one should also account for possible paths. How to handle other coverage criteria is discussed in Section 6.2.3.

\subsubsection{Definition-Use Coverage (DUC)}

Given a service composition $P \equiv \oplus\left(P_{1}, P_{2}, \ldots, P_{n}\right)$ and a variable $x_{j}$, let $a$ be a task that assigns a value to $x_{j}$ and $b$ be a task or a transition that reads the value of $x_{j} .(a, b)$ is a definition-use (DU) pair of $x_{j}$, denoted as $D U\left(x_{j}, a, b\right)$, if and only if there exists a sequence of state transitions in $P$, $s_{0} \stackrel{t_{1}}{\rightarrow} s_{1} \cdots \stackrel{t_{k}}{\rightarrow} s_{k}$, where

- $t_{1}=\left(s_{0}, g_{1}, a_{1}, s_{1}\right), t_{k}=\left(s_{k-1}, g_{k}, a_{k}, s_{k}\right), a=a_{1}, b \in\left\{g_{k}\right.$, $\left.a_{k}\right\}$, and

- $\forall t_{i}=\left(s_{i-1}, g_{i}, a_{i}, s_{i}\right), 1<i<k, a_{i}$ does not assign any value to $x_{j}$.

For example, as illustrated in Fig. 4, there is a DU pair for variable $y$ between $t_{10}$ and $t_{11}$ because $y$ is assigned a value by the task inside $t_{10}$ and the value is read by the guarded expression inside $t_{11}$. Similarly, variable $z_{1}$ is assigned a value by $t_{11}$ and the value is sent to $P_{2}$ via $t_{13}$ and $t_{20}$, and finally read by the guarded expression inside $t_{22}$. Therefore, there is a DU pair for variable $z_{1}$ between $t_{11}$ and $t_{22}$.

The DU coverage criterion tracks all the DU pairs of variables in a service composition and counts how many of them are covered in testing (also referred to as All-uses [83]). That is, let $S_{D U}$ represent the set of all DU pairs for variables and $S_{\text {covered }} \subseteq S_{D U}$ be the set of DU pairs being covered in testing, then the DU coverage adequacy for the test run is equal to $\left|S_{\text {covered }}\right| /\left|S_{D U}\right|$.

\subsubsection{Path Coverage (PC)}

According to Definition 4 , given a service composition $P \equiv$ $\oplus\left(P_{1}, P_{2}, \ldots, P_{n}\right), P_{i} \equiv\left(S_{i}, s_{i, 0}, G_{i}, C_{i} \cup I_{i}, T_{i}, F_{i}\right), i=1 . . n$, a complete path of $P$ is a sequence of state transitions from its initial state to its final state, that is, $s_{0} \stackrel{t_{1}}{\rightarrow} s_{1} \underset{t^{\prime}}{\rightarrow} \stackrel{t_{k}}{\rightarrow} s_{k}$, where $s_{0} \equiv\left(\left(s_{1,0},\{\}\right), \ldots,\left(s_{n, 0},\{\}\right)\right)$ and $\neg \exists s_{k}^{\prime}, s_{k} \stackrel{t_{k}^{\prime}}{\rightarrow} s_{k}^{\prime}$.

The path coverage criterion is to measure the percentage of all the complete paths in a service composition that have been traversed in testing. However, a service composition may have an infinite number of complete paths if there are loops in the service composition. Therefore, in practice, if a complete path is longer than a given maximum length (i.e., the number of state transitions from the initial state is larger than a given maximum number), only the given number of state transitions from the initial state is counted for this path. With this constraint, let $S_{\text {path }}$ be the set of paths of $P$

9. $a_{i}$ can be a task sending $x_{j}$ or a task receiving $x_{j}$. 
that satisfy the above requirements and let $S_{\text {covered }} \subseteq S_{\text {path }}$ be the set of paths that are covered in testing, then the test adequacy for path coverage is equal to $\left|S_{\text {covered }}\right| /\left|S_{\text {path }}\right|$.

\subsection{Event Exposure}

Given a service composition involving services $P_{i} \equiv\left(S_{i}\right.$, $\left.s_{i, 0}, G_{i}, C_{i} \cup I_{i}, T_{i}, F_{i}\right), \quad i=1 . . n$, and the aforementioned structure-based coverage criteria, service providers need to: 1) determine what kinds of events they should advertise in the event interfaces, and 2) publish the event interfaces to service consumers and expose the advertised events in testing. In the following sections, we illustrate how to do that.

\subsubsection{Event Interface for DU Coverage Criterion}

The DU coverage criterion is to track all the DU pairs of variables. For each DU pair of a given variable inside a service, the service provider can define one event to signal the update of the variable and another event to signal the reading of the updated value of the variable. The event sequence of these two events then represents a DU of the variable. However, a variable may be defined in one service and used in another service. To represent a DU that cuts across different services (i.e., coupling def-use [15], [41]), each service provider also needs to declare in its event interface how a variable is assigned a value inside its service and how the value is propagated to another service via its communicating port actions. Similarly, the service receiving the value of a variable from another service must also declare how the value is used inside its service.

For example, as illustrated in Fig. 4, variable $y$ is updated by $t_{10}$ and read by $t_{11}$. This DU pair can be represented by the event sequence $e_{10 \rightarrow 11}, e_{11 \rightarrow 12}$. Similarly, the value of variable $z_{1}$ is updated by $t_{11}$ and propagated to $P_{2}$ via $t_{13}$ and $t_{20}$. The propagated value is then used by $t_{22}$. Therefore, the event sequence $e_{11 \rightarrow 12}, e_{12 \rightarrow 14}$ from $P_{1}$ and the event sequence $e_{20 \rightarrow 21}, e_{21 \rightarrow 23}$ from $P_{2}$ can be used to derive the DU pair for $z_{1}$ across $P_{1}$ and $P_{2}$.

To ease the presentation, we denote $D_{x}, U_{x}, S_{x}$, and $R_{x}$ as the event signals updating variable $x$, reading the value of $x$ (e.g., in a guarded expression or a task), sending the value of $x$ via a port action, and receiving the value of $x$ via a port action, respectively. Then, the event interface (denoted as $E I_{i, d u}$ ) of $P_{i}$ can be defined as follows: $E I_{i, d u} \equiv\left\{\left(e_{s_{1} \rightarrow s_{2}}, e_{s_{k-1}, s_{k}}\right)\right\}$, where $\exists s_{1} \stackrel{t_{1}}{\rightarrow} s_{2} \rightarrow \cdots \stackrel{t_{k-1}}{\rightarrow} s_{k}$, and $e_{s_{1} \rightarrow s_{2}} \in\left\{D_{x}, R_{x}\right\}, e_{s_{k-1}, s_{k}} \in\left\{U_{x}, S_{x}\right\}$, and $x$ is not updated by any $t_{g}(1<g<k-1)$.

To summarize, given the event interface $E I_{i, d u}, i=1$..n, service consumers can construct all the DU pairs of a service composition based on event sequences, denoted as $E S_{D U}$. For any $\left(e_{1}, e_{2}\right) \in E S_{D U}$, if and only if it satisfies any of the following conditions:

- $\left(e_{1}, e_{2}\right) \in E I_{i, d u}$ or

- $\exists\left(e_{1}, e_{1}^{\prime}\right) \in E I_{i_{0}, d u},\left(e_{j_{1}}, e_{j_{1}}^{\prime}\right) \in E I_{i_{1}, d u}, \ldots,\left(e_{j_{k}}, e_{j_{k}}^{\prime}\right) \in$ $E I_{i_{k}, d u},\left(e_{2}^{\prime}, e_{2}\right) \in E I_{i_{k+1}, d u}$ such that $e_{1}=D_{x}$, $e_{1}^{\prime}=S_{x}, e_{2}^{\prime}=R_{x}, e_{2}=U_{x}$, and $\forall l: e_{j_{l}}=R_{x} \wedge e_{j_{l}}^{\prime}=S_{x}$.

\subsubsection{Event Interface for Path Coverage Criterion}

According to the path coverage criterion, we need to enumerate all the paths of a service composition based on events. To do so, we can correlate events from different services and reason about the path coverage based on the event sequence coverage criterion.

To represent a path inside a service, the service can advertise two events indicating the first and the last state changes along the path. However, this is not enough because different paths may share the first and last state changes. To differentiate different paths, the service can expose an event whenever its execution picks a branch (e.g., pick activity in BPEL). In addition, service providers also need to specify the temporal orders (i.e., $\ll$ ) between the exposed events in the event interfaces. For example, in Fig. 4, to represent its two paths, $P_{1}$ can advertise four events associated with the following temporal orders: $e_{10 \rightarrow 11} \ll e_{11 \rightarrow 12} \ll e_{14 \rightarrow 15}, e_{10 \rightarrow 11} \ll e_{11 \rightarrow 13} \ll e_{14 \rightarrow 15}$.

Although each service can advertise events to enumerate its own paths, one remaining issue is how to correlate the individual paths from different services to represent a global path for a service composition. To do so, services also need to advertise events indicating the state changes due to their communication. For example, service $P_{1}$ in Fig. 4 can advertise an event $e_{12 \rightarrow 14}$ when $P_{1}$ sends a message to $P_{2}$. Similarly, service $P_{2}$ can advertise an event $e_{20 \rightarrow 21}$ when $P_{2}$ receives the message from $P_{1}$. Such events can then be used to derive the temporal orders between events from different services, as described in Section 5.

Given the aforementioned events and their temporal orders advertised in the event interfaces, service consumers can organize the events from different services into feasible event sequences. According to Definition 4, each identical event sequence then represents a path of the service composition. For example, suppose services $P_{1}$ and $P_{2}$ in Fig. 4 advertise events $e_{10 \rightarrow 11} \ll e_{11 \rightarrow 12} \ll e_{12 \rightarrow 14} \ll e_{14 \rightarrow 15}$ and $e_{20 \rightarrow 21} \ll e_{21 \rightarrow 23} \ll e_{25 \rightarrow 26}$, respectively. Service consumers can organize the events into a feasible event sequence $e_{10 \rightarrow 11}, e_{11 \rightarrow 12}, e_{12 \rightarrow 14}, e_{20 \rightarrow 21}, e_{21 \rightarrow 23}, e_{25 \rightarrow 26}, e_{14 \rightarrow 15}$. This event sequence represents the path along the transitions $s_{10} \stackrel{t_{10}}{\rightarrow}$ $s_{11} \stackrel{t_{11}}{\rightarrow} s_{12} \stackrel{t_{13}}{\rightarrow} s_{14} \stackrel{t_{15}}{\rightarrow} s_{15}$ and $s_{20} \stackrel{t_{21}}{\rightarrow} s_{21} \stackrel{t_{22}}{\rightarrow} s_{23} \stackrel{t_{25}}{\rightarrow} s_{25} \stackrel{t_{27}}{\rightarrow} s_{26}$ in the service composition. In this way, service consumers can measure the path coverage of a service composition based on the event sequence coverage criterion.

To summarize, service $P_{i}$ can define an event interface for the path coverage criterion (denoted as $E I_{i, p c}$ ) formally as follows: $E I_{i, p c} \equiv\{E S, T O\}$, where $E S \equiv\left\{e_{s_{k} \rightarrow s_{j}}\right\}$ is the set of advertised events satisfying any of the following conditions:

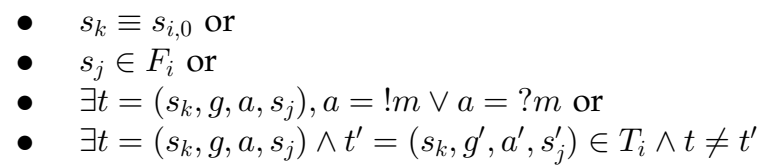

and $T O=E S \times E S$ represents the temporal orders between the advertised events.

\subsubsection{Handling Other Coverage Criteria}

In this paper, we restrict our discussions to the above two structure-based coverage criteria for the following reasons.

First, the focus of this paper is not to investigate and compare the advantages and disadvantages of different structure-based coverage criteria for SOA testing. Instead, we focus on how to reason about the structure coverage of a service composition. We propose two event-based coverage 
criteria (i.e., ECC and ESCC) and use them to reason about the structure coverage of a service composition based on exposed events. Therefore, we use the two representative structurebased coverage criteria for illustration purposes, only.

Second, other control-flow and data-flow coverage criteria can be handled in a similar manner. For example, to reason about the branch or statement coverage [83] of a service composition, service providers can expose events to indicate the execution of a branch or a statement. Then, service consumers can apply the ECC criterion to reason about its coverage based on the exposed events. Similarly, to reason about the all DU path coverage [83] of a service composition, service providers can publish an event interface similar to that for the path coverage. In this way, service consumers can construct a unique set of feasible event sequences, each of which is mapped to a DU path, and apply the ESCC criterion to reason about the all DU path coverage.

In addition, the round-trip strategy is usually applied to measure the test adequacy of a state chart or an objectoriented program [13]. To apply this strategy, service consumers only need to construct and prune the event sequences that can be mapped to the round-trip paths based on the event interfaces for path coverage. Other strategies such as selecting the subset of paths covering loops at least once or selecting linearly independent paths [53] can be handled in a similar way.

\subsubsection{Algorithm for Deriving Event Interfaces}

Above, we presented a formal definition of an event interface for a service with respect to each given coverage criterion. Here, we present an algorithm to derive the event interface.

Algorithm 1 uses a breadth-first search strategy to traverse the service and generate the events for each state change inside a service. A queue (i.e., $q_{\text {search }}$ ) is used to store the states that have not been visited. To track the temporal order between two events for the PC coverage criterion, $E C_{s, \text { from }}$ and $E C_{s, t o}$ are used to cache the events that are raised due to the state changes from state $s$, and the latest events that are raised due to the state changes before state $s$, respectively. Similarly, to keep track of the DU relationship for the DU coverage criterion, $E C_{s, d e f}$ and $E C_{s, \text { use }}$ are used to cache the latest $D_{x}, R_{x}$ events raised before state $s$, and the $U_{x}, S_{x}$ events raised immediately after state $s$, respectively. The algorithm keeps traversing the service until the search queue is empty (Lines 3, 24 to 26). Then, it constructs the temporal orders between events for the PC criterion (Lines 28 to 30) and the DU relationship for the DU coverage criterion (Lines 31 to 33 ).

During the traversal of each transition (Lines 5 to 23), the function GenerateEvents at Line 6 generates all the events (e.g., variableRead events, variableModification events, or more general events as defined in Definition 3) due to a state transition from $c s$ to $s$. Then, the function needExposure at Line 7 filters other events and keeps only the ones that need to be advertised in the event interface in the set $E P$ based on the conditions specified in the above discussion. For the path coverage criterion, if $E P$ is not empty, $E P$ is cached in $E C_{c s, f r o m}$ and $E C_{s, t o}$ (Lines 13 and 14); otherwise, $E C_{c s, t o}$ is propagated to $E C_{s, t o}$ (Line 10). Similarly, for the
DU coverage criterion, whenever an event in $E P$ signals the definition or receiving of a variable (Line 17), this event is put into $E C_{s, d e f}$ (Line 18); whenever an event in $E P$ signals the reading or sending of a variable (Line 19), this event is put into $E C_{c s, u s e}$ (Line 20); if an event from $E C_{c s, d e f}$ signals the definition or receiving of a variable whose value is not updated during the transition (Line 22), then this event is propagated to $E C_{s, d e f}$ (Line 23).

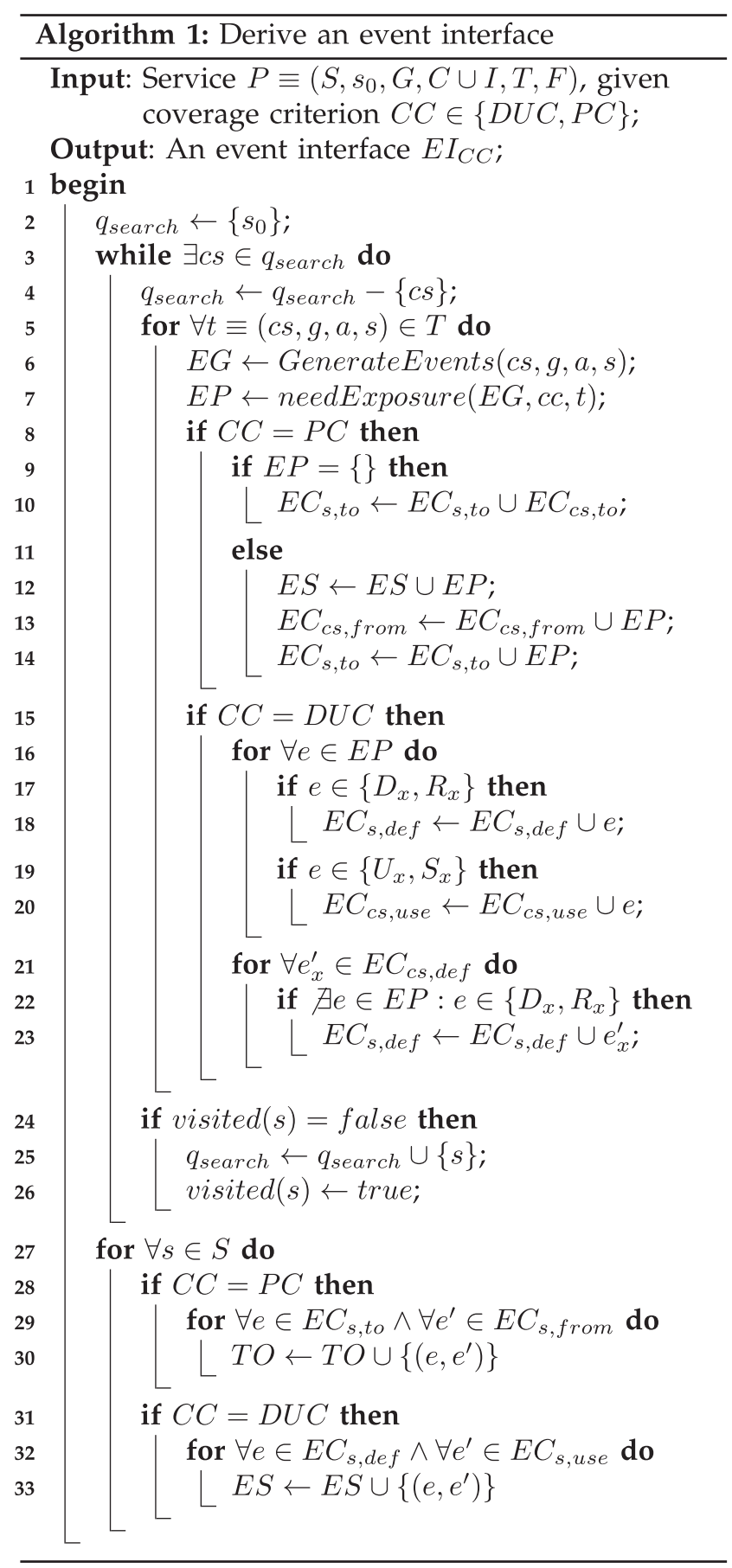

Suppose a service has $k$ transitions and exposes $m$ events in the event interface, then the algorithm traverses the service in at most $k$ steps. To construct the temporal orders between advertised events, the algorithm needs to execute the code at Line 30 or Line 33 at most $m^{2}$ times. Therefore, the complexity of Algorithm 1 in the worst case is $O\left(k+m^{2}\right)$. 


\subsection{Correctness}

Given the aforementioned event interfaces of involved services and the events exposed at runtime, service consumers can reason about the test coverage of a service composition in testing. The test coverage measurement calculated based on exposed events is exactly the same as the test coverage of the service composition. The correctness of this fact is guaranteed by the following theorem.

Theorem 1. Given any of the above two coverage criteria and corresponding event interfaces, the test coverage calculated based on events in testing is exactly the same as the test coverage of a service composition under the given coverage criterion.

The proof of Theorem 1 is to construct a one-to-one mapping between the items that want to be covered (i.e., DU pairs or paths) inside a service composition and the events (or event sequences) advertised in the event interfaces. Details can be found in the Appendix.

With Theorem 1, service consumers can determine how adequately a service composition has been covered in testing. This requires service providers to agree to expose the selected events in the event interfaces. However, due to business interests such as prevention of information leakage, service providers may not be willing to disclose too many details about their internal state changes. In the next section, we illustrate how to address this concern based on event encapsulation.

\section{INFORMATION HiDING}

Although the event interfaces derived in Section 6.2 expose only a subset of events, these events may still reveal information about the implementation details of a service. In this section, we apply information theory to analyze the information leakage from Web services due to event exposure. We then illustrate how to encapsulate the exposed events to reduce information leakage.

According to information theory [66], given a channel with input $X$ and output $Y$, the information of $X$ leaked by $Y$ can be measured as the mutual information between $X$ and $Y$ [22], [51], denoted as $I(X ; Y)$, where $I(X ; Y) \equiv$ $H(X)-H(X \mid Y)$ and $H(X)$ is the entropy of $X$, i.e.,

$$
\begin{aligned}
H(X) & \equiv-\sum_{x \in X} p(x) \log p(x), \\
H(X \mid Y) & \equiv-\sum_{y \in Y} p(y) \sum_{x \in X} p(x \mid y) \log p(x \mid y),
\end{aligned}
$$

and $p(x)$ represents the probability of $x$. The smaller $I(X ; Y)$ is, the less information is leaked. When $X$ and $Y$ are independent, $I(X ; Y)=0$.

The event exposure from Web services can be seen as a channel, where the input $X$ represents the implementation details of a service and the output $Y$ represents the set of exposed events. According to Definition 2, a service consists of states and transitions. Therefore, the potential information leakage of a service's implementation includes state information (i.e., variables and their values) and transition information (i.e., tasks and control flow information).

To address the amount of information leaked via event exposure, this section presents an approach to hide information about the service implementation based on event encapsulation. Informally speaking, event encapsulation means to abstract the necessary information and filter other irrelevant details from events. The formal definition is as follows.

Definition 6 (Event Encapsulation). Given an event $e \equiv$ $\left\{\left(x_{1}, t p_{x 1}, v_{x 1}\right), \ldots,\left(x_{n}, t p_{x n}, v_{x n}\right)\right\}$, event $e^{\prime} \equiv\left\{\left(y_{1}, t p_{y 1}\right.\right.$, $\left.\left.v_{y 1}\right), \ldots,\left(y_{m}, t p_{y m}, v_{y m}\right)\right\}$ is an encapsulation of $e$, denoted as enp $\left(e^{\prime}, e\right)$, if and only if there exists a mapping function $f_{e \rightarrow e^{\prime}}$ such that $\forall V \equiv\left(x_{i}, t p_{x i}, v_{x i}\right) \in e, f_{e \rightarrow e^{\prime}}(V) \in e^{\prime}$.

Based on the concept of event encapsulation, we now illustrate how to hide state information and transition information from events, respectively.

\subsection{State Information Hiding}

Since an event reveals a state change inside a service, service consumers might be able to deduce internal state changes of a service based on the service's exposed events. Let $E$ be the set of all potential internal state changes and $E P$ be the set of exposed events; according to information theory, the amount of such information leakage can be quantified as $I(E ; E P)$.

According to Definition 6, service providers can encapsulate a given event's variables and values into another event whose variables and values have no semantic implications to the former. Then, those encapsulated events, instead of the original events, are advertised and exposed to service consumers. For example, suppose a bookshop service wants to expose an event $e=\left\{\left(x_{\text {stock }}\right.\right.$, int,$\left.v_{\text {stock }}\right),\left(x_{\text {sale }}\right.$, int,$\left.\left.v_{\text {sale }}\right)\right\}$ for testing purposes. To hide the stock and sale information, the service provider can encapsulate this event into another one, $e^{\prime}=\left\{\left(y\right.\right.$, int,$\left.\left.v_{y}\right)\right\}$, where variable $y$ and its value has no physical meaning (and the mapping is invisible to service consumers). In this way, $e$ and $e^{\prime}$ can be seen as independent and the information leakage $I(E ; E P)$ is close to 0 .

This solution protects the state information from being leaked by encapsulating the event variables and values, which remain invisible to service consumers. Although the encapsulated events can be used to reason about the test coverage of a service composition under test, they provide no useful information to derive the test cases. Therefore, a better solution is to encapsulate a subset of events and keep the others $E P^{\prime} \subset E P$, where the event contents in $E P^{\prime}$ are used for deriving test cases (discussed in Section 8). According to information theory, $I\left(E ; E P^{\prime}\right) \leq I(E ; E P)$ : The fewer events are exposed in $E P^{\prime}$, the less information is leaked.

\subsection{Transition Information Hiding}

Since an event reveals only a state transition of a service, the internal task information is kept invisible to service consumers. Therefore, we only need to consider the potential control flow information leakage. According to Definition 2, a service can be regarded as a directed graph with states as nodes and transitions as edges. Service consumers might be able to guess the structure of a service's graph based on the exposed events. Let $G$ represent the graph of a service and EP be the set of exposed events; according to information theory, the amount of such information leakage can be quantified as $I(G ; E P)$. 

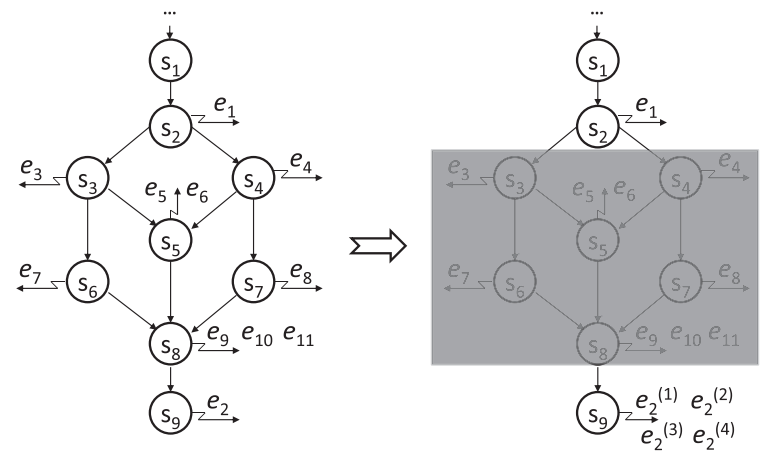

Fig. 5. Encapsulation of event sequences.

In the event interface for the DU coverage criterion, given a DU pair of a variable, service consumers are less likely to deduce the service structure between the points where the variable is defined and used due to the existence of an infinite number of potential paths between the DU pair. However, in the event interface for the path coverage criterion, the temporal order of advertised events is also exposed. Service consumers can still infer the transition information inside a service based on the temporal order of exposed events. In this section, we illustrate how to eliminate transition information leakage from event interfaces for the path coverage criterion.

Our solution is based on filtering out some events from the event interface and encapsulating the temporal orders of all events into the remaining events. We choose to keep only three classes of events in the event interface, that is, $E S^{\prime} \equiv\left\{e_{s_{k} \rightarrow s_{j}}\right\}$, where $e_{s_{k} \rightarrow s_{j}}$ satisfies any of the following conditions:

- $s_{k} \equiv s_{i, 0}$ or

- $s_{j} \in F_{i}$ or

- $\exists t=\left(s_{k}, g, a, s_{j}\right), a=! m \vee a=? m$.

The first two classes of events represent the first and the last state changes along a path inside the service. The third class represents that the service interacts with other services (i.e., sends or receives a message).

The reason we keep these three classes of events and filter all other events is that we can construct an event interface equivalent to the original one in terms of coverage reasoning. To do so, we encapsulate all possible original event sequences based on the remaining events. Given any two events $e_{1}, e_{2} \in E S^{\prime}: e_{1} \ll e_{2} \wedge\left(\neg \exists e_{3} \in E S^{\prime}: e_{1} \ll e_{3} \wedge\right.$ $\left.e_{3} \ll e_{2}\right)$, suppose there are $n$ event sequences between $e_{1}$ and $e_{2}$ (i.e., $e_{1} \ll \cdots \ll e_{2}$ ) in the original event interface, then our solution is to add one variable into $e_{2}$. The added variable is assigned a value from 1 to $n$, each of which corresponds to an event sequence between $e_{1}$ and $e_{2}$.

For example, as illustrated in Fig. 5, suppose only $e_{1}$ and $e_{2}$ need to be exposed. There are four different event sequences from $e_{1}$ to $e_{2}$. To hide the control flow information and internal structure of the service, $e_{2}$ is complemented with an additional variable with the value equal to $1,2,3$, or 4 . The resulting events $e_{2}^{(1)}, e_{2}^{(2)}, e_{2}^{(3)}$, and $e_{2}^{(4)}$ encapsulate the corresponding four event sequences between $e_{1}$ to $e_{2}$.

To reason about the test coverage based on the revised event interfaces, we need to enumerate the possible original event sequences based on the remaining events in the revised event interfaces. To ease the presentation, we denote $e_{2}$ with the value of the added variable equal to $j$ as $e_{2}^{(j)}$. Then we can enumerate the $n$ event sequences between $e_{1}$ and $e_{2}$ as $e_{1} \ll e_{2}^{(j)}(j=1 . . n)$. In the event sequence coverage criterion, we treat $e_{1} \ll e_{2}^{(i)}$ and $e_{1} \ll e_{2}^{(j)}$ $(i \neq j)$ as different event sequences. In this way, the test coverage calculated based on the revised event interface is the same as that using the original event interfaces.

This solution preserves the accuracy of test coverage reasoning and hides transition information inside a service. Since the events representing service interactions and their temporal orders are publicly visible to service consumers based on their service choreography, little information inside a service is leaked from these events. The only information leakage from the revised event interface is the total number of paths inside the service between two temporally consecutive events $e_{1}$ and $e_{2}$. Service consumers, however, are less likely to infer the transition information inside the service based on such information. The reason for this is as follows.

Suppose $e_{2}$ encapsulates $n$ event sequences between $e_{1}$ and $e_{2}$, meaning that there are $n$ possible paths between $e_{1}$ and $e_{2}$ inside the service. Suppose there are a total of $m$ transitions (which are unknown to service consumers) between the state where $e_{1}$ is raised to the state where $e_{2}$ is raised. Then, there are a total of $N=\sum_{i=1}^{m} \prod_{j=m-i+1}^{m} j$ paths (could be more, if a transition exists more than once in a path) between these two states. Therefore, the possibility of inferring the structure of a service implementation between $e_{1}$ and $e_{2}$ is less than $\frac{n !(N-n) !}{N !}$. When $n=2$ and $m=3$, the probability is less than 1 percent, implying that a service's structure and the number of observed paths are nearly independent. Therefore, the transition information leakage $I(G ; E P)$ is small.

\section{Test Case Generation}

As mentioned in Sections 5 and 6, service consumers can subscribe to the events advertised by service providers in their event interfaces and calculate the test coverage based on exposed events from involved services. In this way, service consumers can reason about how well a service composition has been tested.

One important issue remaining is how to design test cases to cover the advertised events or event sequences effectively, that is, how to generate the test cases such that the advertised events or event sequences can be observed in testing under these test cases. In this section, we investigate several different solutions to this issue. The test oracle issue is out of the scope of this paper.

\subsection{Random Testing}

One simple solution is to apply a random testing approach [52] and generate test cases randomly. This solution is simple and no prior knowledge about the service implementation is needed. However, as mentioned in Section 1, the execution of one scenario (e.g., a path) in a service may depend on the execution state of some particular scenarios in another service [13], [72]. A dependence such as this increases the difficulty in effectively generating test cases that cover a service composition adequately. As a result, to 
cover a service composition adequately, service consumers may need to try a large number of test cases (and may thus need a large number of test oracles). This significantly increases the testing effort.

\subsection{Constraint-Based Test Case Generation}

We now illustrate another solution that explores the dependence information among services and attaches this information to event interfaces. The purpose of this is to allow service consumers to use the additionally exposed information to generate test cases effectively to cover a service composition.

To address the difficulty encountered in random test case generation, one natural solution is to explore more information related to exposed events to provide more insights about the internal execution of a service. In particular, we can analyze the service to determine the conditions under which an event can be raised. For example, as illustrated in Fig. 4, event $e_{12 \rightarrow 14}$ is raised if and only if service $P_{1}$ transitions from $s_{10}$ to $s_{14}$, that is, $s_{10} \stackrel{t_{10}}{\rightarrow} s_{11} \stackrel{t_{11}}{\rightarrow} s_{12} \stackrel{t_{13}}{\rightarrow} s_{14}$. Since the guarded condition of $t_{10}$ is always true, $P_{1}$ can transition from $s_{10}$ to $s_{11}$. According to the action in $t_{10}$, state $s_{11}$ should satisfy the following condition: $y=x+10$. If $s_{11} \stackrel{t_{11}}{\rightarrow} s_{12} \stackrel{t_{13}}{\rightarrow} s_{14}$, then $s_{11} \vdash g_{11} \wedge$ $s_{12} \vdash g_{13}$ should be satisfied, that is, the condition ( $y=$ $x+10) \wedge(y<20)$ should be satisfied at state $s_{11}$. Therefore, under this condition, $e_{12 \rightarrow 14}$ is raised to follow after $e_{10 \rightarrow 11}$. To ease the presentation, we call this condition the causality condition between events $e_{10}$ and $e_{11}$, denoted as $C C\left(e_{10}, e_{11}\right) \cdot{ }^{10}$

Note that causality conditions between events can be derived during the construction of event interfaces with Algorithm 1. Formally, suppose $s \stackrel{t}{\rightarrow} s^{\prime}$ and $S C(s)$ represens the constraints $s$ satisfies, then $S C\left(s^{\prime}\right) \equiv S C(s) \wedge g \wedge a$, where $g$ and $a$ are the guarded condition and action in $t$, respectively. ${ }^{11}$ We can iteratively apply this rule during the traversal of a service in Algorithm 1 to calculate the causality conditions between every exposed event and the event that directly precedes it. Suppose event $e_{s_{1} \rightarrow s_{2}}$ directly precedes $e_{s_{k} \rightarrow s_{k+1}}$, that is, $\exists s_{1} \stackrel{t_{1}}{\rightarrow} s_{2} \stackrel{t_{2}}{\rightarrow} \cdots \stackrel{t_{k}}{\rightarrow} s_{k+1}$ and no event is raised during $s_{2} \stackrel{t_{2}}{\rightarrow} s_{3} \stackrel{t_{3}}{\rightarrow} \cdots \stackrel{t_{k-1}}{\rightarrow} s_{k}$. Then we have

$$
C C\left(e_{s_{1} \rightarrow s_{2}}, e_{s_{k} \rightarrow s_{k+1}}\right) \equiv S C\left(s_{1}\right) \wedge\left(\wedge_{j=1}^{k}\left(g_{j} \wedge a_{j}\right)\right),
$$

where $g_{j}$ and $a_{j}$ are the guarded condition and action in transition $t_{j}$, respectively.

By exposing causality conditions between advertised events into event interfaces, service consumers can derive the conditions under which each advertised event can be exposed or each event sequence can be observed. Formally, suppose under condition $c_{1}$ event $e_{1}$ is raised and $e_{1}$ precedes $e_{2}$, then $e_{2}$ is raised under the condition $c_{2} \equiv c_{1} \wedge C C\left(e_{1}, e_{2}\right)$. Similarly, given a feasible event sequence $e_{1}, e_{2}, \ldots, e_{m}$, we can derive the constraint $c$ under which the event sequence can be observed, that is,

10. Note that causality conditions are not restricted to arithmetic conditions. They may include other constraints enforced at the semantic level using first-order logic, which can be handled using SMT solvers (e.g., STP [35]).

11. We can always rename the variables to make sure each variable is assigned a value only once. The assignment operator " $:=$ " in an action is then replaced by the equal operator " $="$ in the expression. Therefore, we can calculate the constraint of $S C\left(s^{\prime}\right)$ in this way. $c \equiv c_{1} \wedge\left(\wedge C C\left(e_{j}, e_{k}\right)\right) \quad(1 \leq j, k \leq m)$, where $e_{j}$ directly precedes $e_{k}$.

For example, given a feasible event sequence $e_{10 \rightarrow 11}$, $e_{11 \rightarrow 12}, e_{12 \rightarrow 14}, e_{20 \rightarrow 21}, e_{21 \rightarrow 23}, e_{23 \rightarrow 25}, e_{25 \rightarrow 26}, e_{14 \rightarrow 15}$ in Fig. 4 and the causality conditions between these events, the constraint for this event sequence is equal to $(y=x+$ 10) $\wedge(y<20) \wedge\left(z_{1}=2 y\right) \wedge\left(z_{1}<30\right) \wedge\left(u_{1}=z_{1}-6\right) \wedge(w=$ $\left.u_{1}+2\right)$. When the constraint is satisfied, this event sequence can be observed. By applying constraint solving techniques to this constraint, service consumers can obtain a solution $\left\{x=2, y=12, z_{1}=24, u_{1}=18, w=20\right\}$. This solution indicates a test case (that is, $\{x=2\}$ ) to cover the feasible event sequence.

This solution is effective in generating test cases to cover the advertised events or event sequences, but it suffers from two limitations: 1) The exposed causality conditions cause information leakage from a service implementation; 2) the complexity of constraint solving is high when the number of variables is large. In the next section, we propose a hybrid solution to combine random testing with constraintbased test case generation. The purpose is to trade off the effectiveness of test cases, efficiency of test case generation, and information leakage.

\subsection{Hybrid Solution}

In random testing, test cases are generated randomly. No prior knowledge about the service implementation is needed. Therefore, test cases can be generated efficiently and no information is leaked. The limitation of random testing is that a large number of test cases may be needed to adequately cover a service composition. On the other hand, constraint-based test case generation is effective in covering a service composition but suffers from information leakage and high complexity of test case generation. Therefore, we consider combining both solutions to trade off the effectiveness of test cases, efficiency of test case generation, and information leaked.

Our basic idea is to relax the constraints for events or event sequences. Instead of generating a constraint for every individual event or event sequence, we combine the constraints for a group of different events or event sequences into one constraint. To further hide the service implementation details, the resulting constraint can be relaxed to include some test cases that do not cover the given events or event sequences in the group. Next, we randomly generate test cases and filter test cases based on the constraint. The randomly generated test cases are filtered if they do not satisfy the constraint. In this way, we avoid the high complexity of applying constraint solving to generate each test case. More importantly, compared to random testing, the test cases generated are more effective. On the other hand, less information is leaked because the exposed constraints are relaxed.

In general, a more relaxed constraint (which means it has a large solution space) implies that: 1) Less information is leaked (because it is less specific about the service implementation and more variables are hidden), and 2) the generated test cases are less effective in covering the events or event sequences (because more test cases that do not cover the events or event sequences are also included), and 3) the test case generation is more efficient 


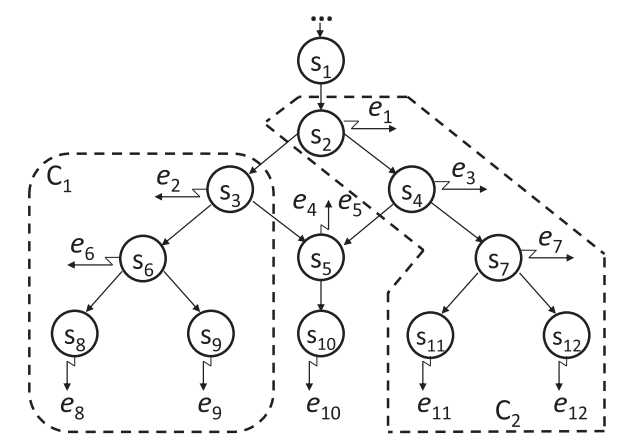

Fig. 6. Hybrid test case generation.

(because randomly generated test cases are less likely to be filtered by a constraint with a large solution space). Therefore, service providers can trade off among the effectiveness of test cases, information leakage, and efficiency of test case generation by controlling the granularity of the constraints exposed.

Given a set of events $e_{1}, e_{2}, \ldots, e_{n}$ and the corresponding conditions $C_{1}, C_{2}, \ldots, C_{n}$ under which the events can be generated. A simple way to generate a single constraint for this set of events is to combine all the conditions into one $C_{1} \cup C_{2} \cup \ldots \cup C_{n}$. This solution, however, needs to derive $C_{i}$ for each event $e_{i}$ first.

Another solution is to determine the event $e_{i}$ that precedes all the other events, that is, $\forall j, j \neq i, e_{i} \ll e_{j} . C_{i}$ is then exposed as the relaxed constraint for the set of events. ${ }^{12}$ For example, as depicted in Fig. 6, the condition under which $e_{6}$ is raised is used to represent the relaxed constraint for events $e_{8}$ and $e_{9}$. Similarly, for a set of event sequences, we can find their shared prefix and use the conditions for the shared prefix to represent the relaxed constraint for the set of event sequences. For example, the relaxed constraint for the event sequences $e_{1}, e_{3}, e_{7}, e_{11}$ and $e_{1}, e_{3}, e_{7}, e_{12}$ in Fig. 6 can be derived as the conditions for the event sequence $e_{1}, e_{3}, e_{7}$.

\section{Evaluation}

As illustrated in Sections 6 and 8, our approach makes use of events exposed by services via their event interfaces to determine the test coverage of an entire service composition and to derive test cases for the service composition. This section evaluates our approach quantitatively by comparing it to existing work in terms of test coverage rate, effectiveness in fault-detection, and test case generation. We also analyze and evaluate the tradeoff between information leakage and effectiveness of test case generation. Finally, we evaluate the running time complexity of our algorithms and the overhead of event exposure.

\subsection{Evaluation of Test Coverage and Effectiveness of Fault Detection}

\subsubsection{Experimental Setup}

We use five open-source service compositions to evaluate our work: A loan approval application [40] (denoted as LA), a book ordering application [61] (denoted as BO), a

12. In the evaluation, we use the latter approach as our hybrid solution.
TABLE 1 Applications and Descriptive Statistics

\begin{tabular}{|c|c|c|c|c|}
\hline Application & \#service & \#activity & \#transition & \#line \\
\hline$L A$ & 4 & 28 & 24 & 630 \\
\hline$B O$ & 3 & 29 & 37 & 750 \\
\hline$S C$ & 3 & 30 & 33 & 758 \\
\hline$I N$ & 6 & 52 & 58 & 961 \\
\hline$A C$ & 12 & 96 & 117 & 2614 \\
\hline
\end{tabular}

supply-chain application [74] (denoted as SC), an insurance claim application [79] (denoted as IN), and an auction application [37] (denoted as AC). Each application is characterized in Table 1. These applications have also been widely used for service testing and verification in the literature [8], [42], [55], [79], [81].

In this experiment, we evaluate the coverage percentage in testing and the effectiveness in fault detection of our approach (denoted as OA). We adapt the approach proposed by Bartolini et al. [4] as a baseline (denoted as EA). That is, given a composition of services $s_{1}, s_{2}, \ldots, s_{n}$, and letting $c_{i}$ be the coverage of $s_{i}$ reported by the approach [4], EA measures the average coverage of this service composition as $c=\sum_{i=1}^{n} c_{i} / n$.

Faulty versions of service compositions are needed for the experiment. To the best of our knowledge, few faulty versions of compositions have been reported by developers. Therefore, we generate different faulty versions of service compositions by seeding one fault into the five original service compositions using mutation testing techniques [28]. We systematically generate mutations for the five service compositions: Each faulty version has one mutation. Two types of mutations are generated: Type 1 corresponds to the faults internal to a service and Type 2 represents inconsistency faults that cut across different services in a service composition (e.g., manipulation of the incompatible ISBN numbers by the Catalog WS and the Warehouse WS from Fig. 1 from our earlier example.)

To generate faulty versions of Type 1, we systematically replace the operators, variables, and constants in the guarded expressions and actions with a corresponding mutation. ${ }^{13}$ To generate faulty versions of Type 2 , we systematically add a pair of inconsistent operations (e.g., the total amount for two accounts changed after transferring money from one to the other) into pairs of tasks from different services. The joint execution of both tasks represents an inconsistency fault. To filter equivalent mutants, ${ }^{14}$ we test every faulty version using all the test cases in the test pool. ${ }^{15}$ If a mutant cannot be detected by any test case in the test pool, we regard it as an equivalent mutant and remove it from the set of faulty versions. Finally, we obtain 965 faulty versions of the three service compositions (Type 1: 710, Type 2: 255), a detailed description of the seeded mutations is shown in Table 2.

13. We do not generate mutations for XML data [77]. Instead, we seed mutations for the communication tasks (e.g., replacing the variables in the tasks) to generate invalid message faults.

14. The term equivalent mutant means that the program resulting from applying the mutation operators is equivalent to the original one.

15. We generate 100,000 test cases in the test pool using the random testing approach [52]. 
TABLE 2

Summary of Mutations by Categories

\begin{tabular}{|c|c|c|c|c|c|c|}
\hline \multirow{2}{*}{ Category } & \multicolumn{5}{|c|}{ Service composition } & \multirow{2}{*}{ Sum. } \\
\cline { 2 - 6 } & LA & BO & SC & IN & AC & \\
\hline Operator & 18 & 80 & 125 & 91 & 29 & 343 \\
\hline Variable & 14 & 47 & 104 & 58 & 40 & 263 \\
\hline Constant & 14 & 25 & 16 & 33 & 16 & 104 \\
\hline Inconsistency & 6 & 32 & 20 & 131 & 66 & 255 \\
\hline Sum. & 52 & 184 & 265 & 313 & 151 & 965 \\
\hline
\end{tabular}

We randomly select a test case from the test pool and execute a target faulty version of a service composition over the test case. If the test case improves the coverage percentage reported by OA or EA under a given coverage criterion, $^{16}$ then it is added to the test suite for the corresponding approach. The test case selection procedure terminates if 100 percent coverage is reported by the corresponding approach or if, after a maximum number (200) of trials, the coverage has not improved. This procedure is repeated 128 times for each faulty version.

To compare the test coverage of OA and EA under a given coverage criterion, we measure the real test coverage of the entire service composition in testing using each approach with the aforementioned test suites. To evaluate the effectiveness of fault detection, we measure the faultdetection rate [39]. The fault-detection rate (denoted as fdrate) is calculated as the ratio of the number of test suites that can detect the fault in the faulty version over the total number of test suites selected.

\subsubsection{Experiment Data Analysis}

In this section, we analyze and report the experimental results.

To compare the test coverage of OA and EA for each given coverage criterion $X(X \in\{D U C, P C\})$, we test the following hypothesis:

- $H_{0}$ (null hypothesis). Given a coverage criterion $X \in\{D U C, P C\}$, there is no significant difference between the real test coverage using OA and EA.

- $H_{1}$ (alternative hypothesis). Given a coverage criterion $X \in\{D U C, P C\}$, the real test coverage using $\mathrm{OA}$ is significantly larger than that of EA.

To evaluate the effectiveness of fault-detection, we test the following hypothesis:

- $H_{0}$ (null hypothesis). Given a coverage criterion $X \in\{D U C, P C\}$, there is no significant difference between the fault-detection rate using OA and EA.

- $H_{1}$ (alternative hypothesis). Given a coverage criterion $X \in\{D U C, P C\}$, the fault-detection rate using $\mathrm{OA}$ is significantly larger than that of EA.

In the experiment, we calculate the average real coverage and fault-detection rate for each faulty version using both $\mathrm{OA}$ and EA approaches. The statistics of the real coverage and fault-detection rate for each faulty version using both approaches are shown in Fig. 7. To ease the comparison, we calculate the difference of coverage (and fault detection

16. Note that the coverage percentage reported by OA is given by the formula in Section 5. rate) using $\mathrm{OA}$ and $\mathrm{EA}$, that is, $D I F F_{\text {coverage }}=$ coverage $_{\mathrm{OA}}-$ coverage $_{E A}$ and $D I F F_{\text {fdrate }}=$ fdrate $_{O A}-$ fdrate $_{E A}$.

The distribution of $D I F F_{\text {coverage }}$ using DU and the path coverage criteria for faults of Types 1 and 2 are shown in Figs. $7 \mathrm{a}$ and $7 \mathrm{~b}$, respectively. We can observe that $D I F F_{\text {coverage }}$ shares similar patterns between faults of Types 1 and 2: For both types of faults, $D I F F_{\text {coverage }}$ using the DU coverage criterion varies between $[0,0.15]$ and $D I F F_{\text {coverage }}$ using the path coverage criterion varies between $[-0.1,0.6]$. Fig. $7 \mathrm{c}$ shows the average coverage of OA and EA and the corresponding standard errors: Using the DU coverage criterion, on average OA can achieve 7,8 , and 7 percent more coverage than EA for faults of Type 1, Type 2, and overall, respectively; using the path coverage criterion, on average, OA can achieve 23, 36, and 27 percent more coverage than EA for faults of Type 1, Type 2, and overall, respectively. The detailed improvement of coverage for each application is shown in Table 3 . We find that DIF $F_{\text {coverage }}$ using PC is larger than that using DUC ( 0.27 versus 0.07 on average). This is because the path coverage criterion measures the coverage of global paths only in a service composition, whereas the DU coverage criterion measures both local DU pairs inside a service and the DU pairs across different services. Therefore, compared to the DU coverage criterion, OA (which is from a global perspective) can achieve much higher coverage than EA (which is from a local perspective) using the path coverage criterion.

The distribution of $D I F F_{\text {fdrate }}$ using the DUC and PC coverage criteria for faults of Types 1 and 2 is shown in Figs. 7d and 7e, respectively. For faults of Type 1, $D I F F_{\text {frate }}$ varies mainly between $[0,0.2]$ and $[0,0.1]$ using the DU and path coverage criteria, respectively. For faults of Type 2,DIFF $F_{\text {frate }}$ varies mainly between $[0,0.3]$ and $[0,0.5]$ using the DU coverage and path coverage criteria, respectively. The average fault-detection rate using $\mathrm{OA}$ and EA and corresponding standard errors are shown in Fig. 7f: Using the DU coverage criterion, on average OA achieves a 6, 9, and 7 percent higher fault-detection rate than EA for faults of Type 1, Type 2, and overall, respectively; using the PC coverage criterion, $\mathrm{OA}$ achieves on average a 3, 9, and 5 percent higher fault-detection rate than EA for faults of Type 1, Type 2, and overall, respectively. The detailed improvement of fault-detection rate for each application is shown in Table 3. We can find that the improvement is up to 24 percent for faults of Type 2 and 8 percent for faults of Type 1. We can also observe that compared to faults of Type $1, D I F F_{\text {frate }}$ (using both PC and DUC) is much larger for faults of Type 2. This implies that our approach is more sensitive to detect inconsistency faults across different services.

To confirm our observation and test the above hypothesis, we applied the nonparametric Wilcoxon Signed-Rank Test [73] to verify the statistical significance of our experimental results. We also calculated the HodgesLehmann estimator [38] for the median and the 95 percent confidence interval of the difference $(E A-O A)$. The $p$ values of the tests, difference median, and confidence interval are shown in Table 4. Given a significance level equal to 0.05 , all the null hypotheses are rejected except the one about the fault-detection rate for faults of Type 1 using the path coverage criterion. This confirms our observation from Fig. 7. That is, our approach can achieve a higher 


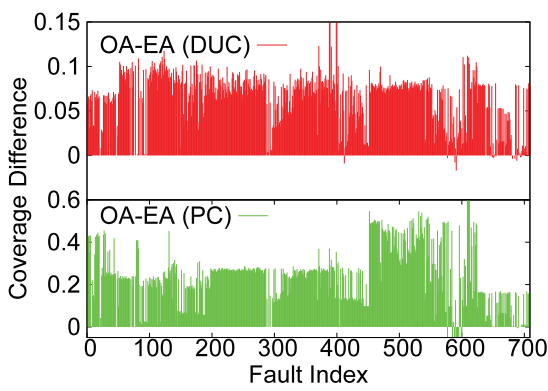

(a) Type 1

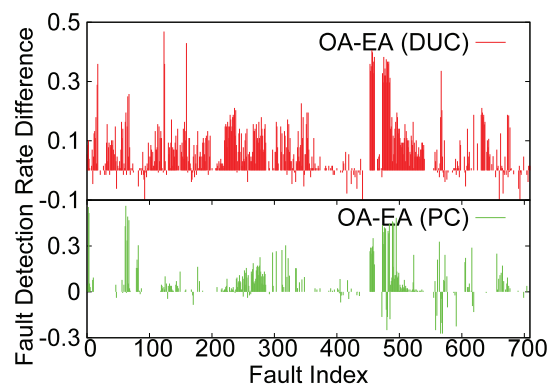

(d) Type 1

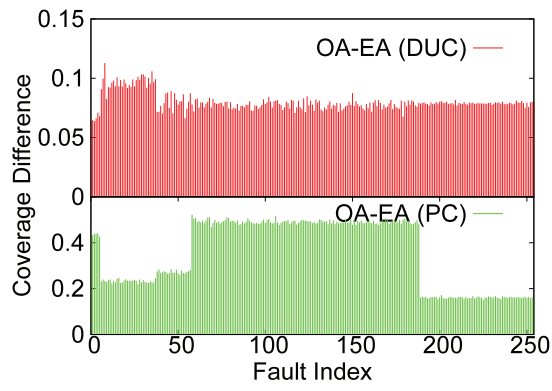

(b) Type 2

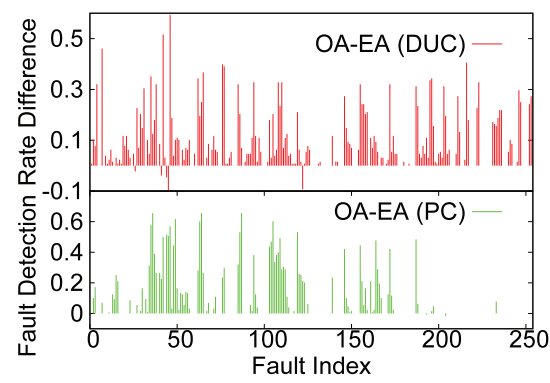

(e) Type 2

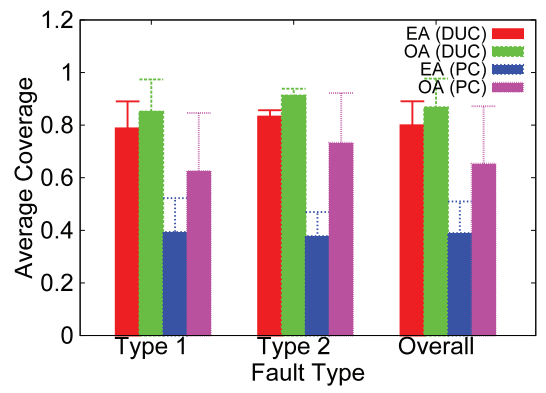

(c) Average

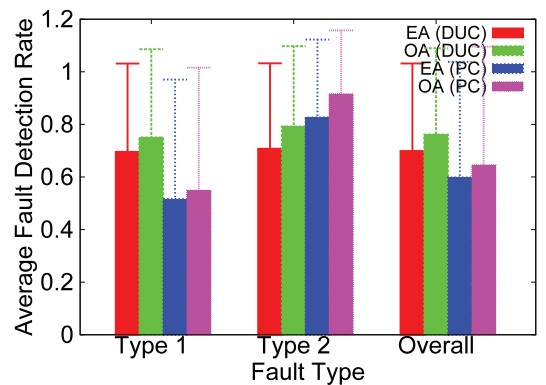

(f) Average

Fig. 7. Statistics about coverage and fault-detection rate.

TABLE 3

Summary of Average Improvements by Applications

\begin{tabular}{|c|c|c|c|c|c|c|c|c|c|c|}
\hline Measurement & \multicolumn{1}{c|}{$D I F F_{\text {coverage }}$} & \multicolumn{1}{c|}{ DIF $F_{\text {fdrate }}$} \\
\hline Application & $L A$ & $B O$ & $S C$ & $I N$ & $A C$ & $L A$ & $B O$ & $S C$ & $I N$ & $A C$ \\
\hline \hline \multicolumn{10}{|c|}{$D U C$} \\
\hline Type 1 & 0.0528 & 0.0733 & 0.0704 & 0.0636 & 0.0355 & 0.0598 & 0.0547 & 0.0456 & 0.0778 & 0.0269 \\
\hline Type 2 & 0.0663 & 0.0971 & 0.0791 & 0.0776 & 0.0790 & 0.0846 & 0.0896 & 0.1055 & 0.0767 & 0.0910 \\
\hline Overall & 0.0544 & 0.0774 & 0.0711 & 0.0695 & 0.0545 & 0.0267 & 0.0608 & 0.0501 & 0.0773 & 0.0550 \\
\hline \hline \multicolumn{10}{|c|}{$P C$} \\
\hline Type 1 & 0.2873 & 0.1841 & 0.2322 & 0.3249 & 0.0838 & 0.0307 & 0.0285 & 0.0285 & 0.0463 & 0.0124 \\
\hline Type 2 & 0.4340 & 0.2330 & 0.2727 & 0.4917 & 0.1613 & 0.0456 & 0.0974 & 0.2375 & 0.1117 & 0.0018 \\
\hline Overall & 0.3042 & 0.1926 & 0.2353 & 0.3947 & 0.1177 & 0.0324 & 0.0405 & 0.0443 & 0.0737 & 0.0078 \\
\hline
\end{tabular}

TABLE 4

P Values of Hypothesis Testing, Difference Median, and 95 Percent Confidence Interval

\begin{tabular}{|c|c|c|c|c|c|c|}
\hline & \multicolumn{3}{|c|}{ Coverage } & \multicolumn{3}{|c|}{ Fault Detection Rate } \\
\hline & $p$ & median & confidence interval & $p$ & median & confidence interval \\
\hline \multicolumn{7}{|c|}{$X=D U C$} \\
\hline Type 1 & $\leq 0.01$ & -0.069 & {$[-0.071,-0.066]$} & $\leq 0.01$ & -0.043 & {$[-0.047,-0.035]$} \\
\hline Type 2 & $\leq 0.01$ & -0.079 & {$[-0.079,-0.078]$} & $\leq 0.01$ & -0.059 & {$[-0.078,-0.047]$} \\
\hline Overall & $\leq 0.01$ & -0.075 & {$[-0.076,-0.073]$} & $\leq 0.01$ & -0.047 & {$[-0.051,-0.039]$} \\
\hline \multicolumn{7}{|c|}{$X=P C$} \\
\hline Type 1 & $\leq 0.01$ & -0.233 & {$[-0.245,-0.222]$} & 0.09 & -0.004 & {$[-0.008,0.000]$} \\
\hline Type 2 & $\leq 0.01$ & -0.350 & {$[-0.363,-0.330]$} & $\leq 0.01$ & -0.031 & {$[-0.059,-0.012]$} \\
\hline Overall & $\leq 0.01$ & -0.258 & {$[-0.268,-0.250]$} & $\leq 0.01$ & -0.008 & {$[-0.012,-0.004]$} \\
\hline
\end{tabular}

coverage and fault-detection rate than the baseline approach. Note that the testing result provides no significant evidence that our approach outperforms the baseline approach in detecting faults of Type 1 using the path coverage criterion. This is because faults of Type 1 are internal to a service, and both our approach and the baseline approach have similar coverage of local paths inside a service. Therefore, in many faulty versions of Type 1, OA and EA have the same fault detection rate. If we exclude the zero in the Wilcoxon Signed-Rank Test (i.e., all faulty versions in both approaches that have same faultdetection rate are not counted and ranked), then the null hypothesis can still be rejected.

\subsection{Evaluation of Test Case Generation}

\subsubsection{Experiment Setup}

In this experiment, we evaluate the effectiveness of test case generation for service compositions using the three 


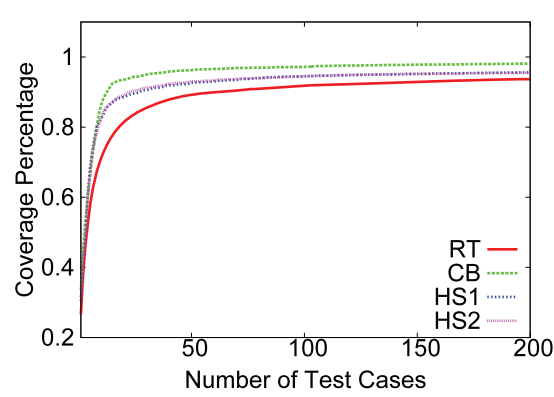

(a) Effectiveness (DUC)

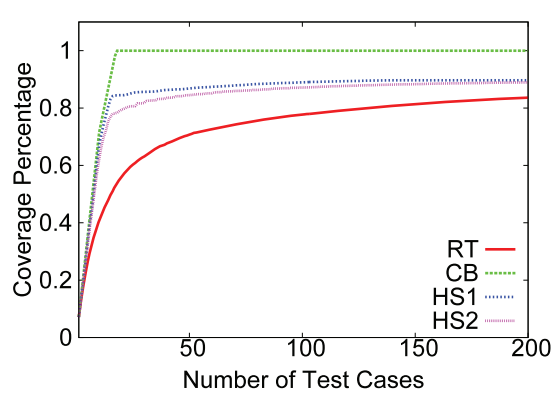

(b) Effectiveness (PC)

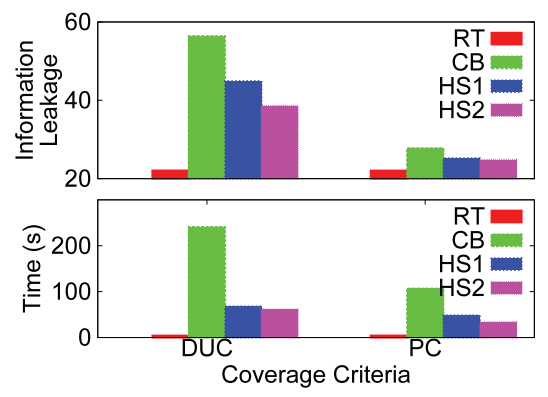

(c) Efficiency and information leakage

Fig. 8. Effectiveness, efficiency, and information leakage of test case generation.

approaches proposed in Section 8: Random testing (denoted as RT), constraint-based test case generation (denoted as $\mathrm{CB}$ ), and hybrid solution (denoted as HS). For the hybrid solution, we group constraints of some events (or event sequences) into one event (or event sequence) and keep $n$ percent of the total constraints. In the experiment, we assign $n$ equal to 75 (denoted as HS1) and 50 (denoted as HS2), respectively. We compare the effectiveness of test case generation for each approach and track the corresponding side effects (i.e., information leakage and time needed for test case generation).

In the experiment, we applied each of the above approaches to generate 200 test cases for each of the service compositions introduced in Section 9.1. We recorded the time needed for generating all the test cases. We then tested the service compositions using the generated test cases and recorded the test coverage of the service compositions and corresponding information leakage. The experiment is repeated 128 times and results are averaged over all runs.

To evaluate the effectiveness of test case generation, we compare the number of test cases needed to cover each service composition with a given coverage percentage requirement (e.g., 90 percent path coverage). The fewer test cases are needed, the more effective the test case generation is. To measure the efficiency of test case generation, we measure the total time needed for generating the 200 test cases. The lower the time, the more efficiently test cases are generated.

In the experiment, we measure the state information leakage only because little transition information is leaked according to the information hiding solution from Section 7. Let $X$ be the set of events generated in the experiment and $Y$ be the set of exposed events. Since all the events in $X-Y$ are invisible to service consumers, we treat them independent of $Y$. That is, service consumers cannot derive these events from observing $Y$. Therefore, $I(X ; Y)=H(Y)=-\sum_{y \in Y} p(y) \log p(y)$. In the experiment, we estimate the value of $p(y)$ as $n / N$, where $n$ is the number of times event $y$ is raised in a total of $N$ runs.

\subsubsection{Experiment Data Analysis}

The experiment results are shown in Fig. 8. Figs. $8 \mathrm{a}$ and $8 \mathrm{~b}$ show the effectiveness of different test case generation approaches under the DU and path coverage criteria, respectively. Among these approaches, $C B$ is the most effective one, in the sense that it can achieve the highest coverage percentage and uses the least number of test cases to achieve a given coverage percentage. On the contrary, RT is the least effective one compared to the others. The effectiveness of HS1 and HS2 lies between CB and RT. For example, Fig. 8a shows that to achieve 90 percent DU coverage, HS1 and HS2 use 80 percent more test cases than $\mathrm{CB}$, but use 55 percent fewer test cases than RT. Note that in the experiment for DU coverage, many DU pairs share the same constraints. As a result, HS1 and HS2 have similar constraints and similar performance.

For the path coverage criterion, HS1 performs better than HS2. For example, as illustrated in Fig. 8b, to cover 85 percent paths, HS1 uses 58 percent fewer test cases than HS2. Moreover, both HS1 and HS2 perform much better than RT: RT uses 200 test cases to achieve 84 percent path coverage, whereas HS1 and HS2 use only 16 and 44 test cases to achieve the same performance. Fig. $8 \mathrm{~b}$ also shows that $\mathrm{CB}$ has better performance than HS1 and HS2 (CB achieves 10 percent more coverage).

Fig. $8 \mathrm{c}$ shows the efficiency and information leakage of all the test case generation approaches. Under both DU and path coverage criteria, $\mathrm{CB}$ is the most time-consuming approach and leaks more information than the others. On the contrary, RT leaks the least information and is the most efficient approach for generating test cases. The efficiency and information leakage of HS1 and HS2 lie between that of $\mathrm{CB}$ and RT, but HS1 needs more time and leaks more information than HS2. Note that for each of the above approaches, the time needed for test case generation and information leakage under the DU coverage criterion are higher than that under the path coverage criterion. This is because more constraints and events are exposed in the event interfaces for the DU coverage criterion.

In summary, the experimental results show that the hybrid test case generation solution trades off effectiveness, efficiency, and information leakage: It is more effective than random test case generation, uses less time, and leaks less information than the constraint-based solution. The results also show that the more information is leaked, the more effectiveness the hybrid solution can achieve, and the more time is needed to generate the test cases. Therefore, the experimental results suggest that service providers can customize the visibility of their event interfaces to help service consumers generate test cases with different quality requirements.

\subsection{Evaluation of Runtime Overhead}

Finally, we evaluate the runtime complexity of our solution. We randomly generated a set of services with the number of 


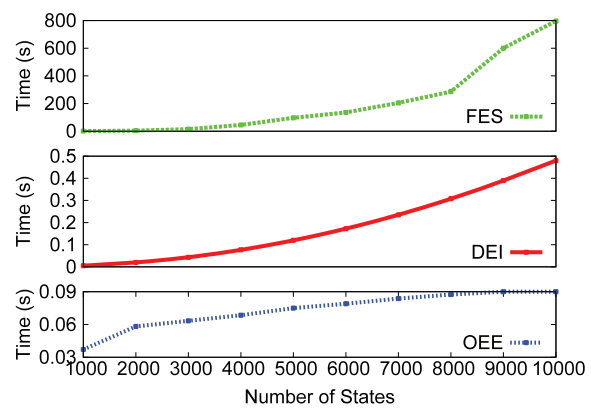

Fig. 9. Runtime overhead.

states varying from 1,000 to 10,000 and applied our algorithm to derive event interfaces (denoted as DEI). The overhead for exposing events at runtime for each service is also recorded (denoted as OEE). We also construct feasible event sequences for randomly generated service compositions with the number of states varying from 1,000 to 10,000 (denoted as FES). The experiment is repeated 1,000 times and the average time needed is recorded.

The experimental results are shown in Fig. 9. The results show that it takes less than 1 second to derive an event interface using Algorithm 1 and less than 0.1 seconds to expose all the declared events at runtime for a service with 10,000 states. For a service composition with 10,000 states, it takes less than 800 seconds to construct all the feasible event sequences. Therefore, the runtime overhead of our solution is small.

In addition, we also record the testing cost in terms of execution time in the experiment. The more test cases are executed, the more execution time is needed. For example, it takes about 3 hours on average to execute 200 test cases for the Auction application in our current experimental setting. When the maximum length of paths in the Auction application is set to be twice the current setting (due to loops), it needs at least 40,000 test cases to well cover all the paths, resulting in about 600 hours for the whole testing. Therefore, service consumers may need to trade off the number of test cases (so as the coverage percentage) and the testing cost when the testing is very expensive.

\subsection{Threats to Validity}

Construct validity. The experimental results may be invalid if concepts were mismeasured using wrong variables. One purpose of our experiments is to evaluate the benefits of our approach, which include accurate coverage reasoning, more adequate testing of a service composition, and effectiveness of test case generation. Therefore, we measured the quantitative benefits of our approach in terms of coverage rate, fault-detection rate, and number of test cases needed.

To measure the information leakage due to event exposure, we apply information theory to calculate the mutual information between the exposed events and the events internal to the service implementation. Mutual information is also applied in other fields to measure information leakage [22], [51]. Besides mutual information, the exposed events may also reveal information to service consumers due to the semantics of these events (e.g., the name of variables, tasks, etc.). We do not measure this kind of information leakage because our solution suggests that service providers encapsulate the exposed events (e.g., renaming the variables) to avoid semantic information leakage.

Internal validity. Confounding factors like the types of seeded faults and test case selections may affect the causeeffect relationships in the experiment if the seeded faults are sensitive to only particular paths or DU pairs across the service composition or the test cases are selected to cover each service well but only cover a few paths in the service composition as a whole. We alleviate the impact of these factors by seeding different types of faults evenly across a service composition using mutation testing techniques and randomly selecting test cases from a large pool of test cases.

In the experiment, we tried a maximum number of 200 test cases for each approach, and each approach is repeated 128 times. We choose 200 test cases because we found that the coverage percentage does not increase significantly even when we try more test cases (e.g., 500) for the applications. Each approach is repeated 128 times because the standard deviation for the average result is small enough (e.g., the standard deviation is less than 1 percent of the average result).

External validity. Another concern is the generalization of the experimental results. We address this issue by using five representative applications and a set of large-scale simulation studies. The five applications are also widely used in existing service testing and verification work [8], [42], [55], [79], [81]. The number of services in the five applications varies from 3 to 12, and the lines of code vary from 700 to 2600. The structure of the involved services includes workflow patterns such as sequence, parallel flow, branch, loop, and so on. Although these applications are of smaller scale, the scale of the applications is not a major confounding factor affecting the experimental results regarding test coverage, fault detection rate, effectiveness of test cases, and information leakage because these metrics are mainly determined by how adequately the applications are covered by the test cases and how events are exposed. On the other hand, we also conduct a set of large-scale simulation studies to evaluate the overhead of our solution, which is affected by the scale of the applications.

Theoretical reliability. Finally, we repeated the experiments many times to remove accidental errors. All the experiment subjects and data are available online ${ }^{17}$ and can be repeated.

\section{Discussion AND Limitations}

Deriving the formal model. In this paper, we model a service as a finite state machine, but our work is not limited to this formal model. In practice, services may be implemented in BPEL and other languages. We can apply many existing approaches to transform BPEL and other services (e.g., Java [24]) into formal models, such as finite state machines [33] and process algebras [32], to just name a few. On the other hand, to derive the causality conditions among events, the semantics of actions are needed. Some existing Web service standards (like OWL-S [69]) provide such semantics for services (e.g., the pre/postconditions). In addition, the formal models of services with the semantics

17. http:/ /sites.google.com/site/cyye76/st.tar. 


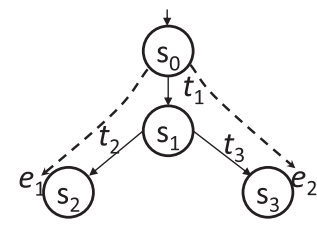

(a)

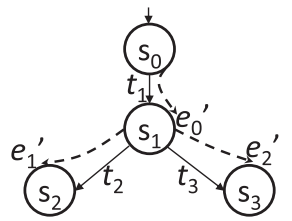

(b)
Fig. 10. Illustration of transition information leakage.

of actions can be derived using symbolic execution techniques [23]. Our work can be applied to the model based on the existing work.

Measurement of information leakage. In this paper, we use mutual information to quantify the leakage of state and transition information due to event exposure. Such measurement is reasonable.

For example, suppose the Bookshop WS in Fig. 1 classifies books into different discount categories based on their prices. How to classify books is the business information of the Bookshop WS and kept invisible to service consumers. To simplify for illustration purposes, we assume variable $\{x$, int, $1 \leq x \leq 100\}$ represents the book price, which satisfies the discrete uniform distribution, and variable $\{y$, int, $1 \leq y \leq 5\}$ represents the category. The Bookshop WS classifies a book with price $x$ to category $y$, satisfying $y=(x-1) / 20+1$. Two events, $e_{x}=$ $\{x$, int, $1 \leq x \leq 100\}$ and $e_{y}=\{y$, int, $1 \leq y \leq 5\}$, can be raised to represent the internal state changes of the service. Now let us consider the following event exposures: $E P_{1}=$ $\left\{e_{x} \mid 1 \leq x \leq 20\right\}, E P_{2}=\left\{e_{x} \mid 1 \leq x \leq 40\right\}$, and $E P_{3}=\left\{e_{x} \mid 1 \leq\right.$ $x \leq 40\} \cup\left\{e_{y} \mid 1 \leq y \leq 3\right\}$. Intuitively, $E P_{1}$ reveals less information and $E P_{3}$ reveals more information than $E P_{1}$ and $E P_{2}$. This can be confirmed by calculating the state information leakage of the three event exposures. According to the above description, we have $p\left(e_{x}\right)=1 / 100$, $p\left(e_{y}\right)=1 / 5, p\left(e_{x} \mid e_{x}^{\prime}\right)=1$ if $x=x^{\prime}$; otherwise $0,\left(e_{y} \mid e_{y}^{\prime}\right)=1$ if $y=y^{\prime}$; otherwise $0, p\left(e_{x} \mid e_{y}\right)=1 / 20$ if $y=(x-1) / 20+1$ 1 ; otherwise $0, p\left(e_{y} \mid e_{x}\right)+1$ if $y=(x-1) / 20+1$; otherwise 0 . Therefore, $I\left(E ; E P_{1}\right), I\left(E ; E P_{2}\right)$, and $\left(E ; E P_{3}\right)$ is equal to $\frac{1}{5} \log 5, \frac{2}{5} \log 5$, and $\log 5$, respectively.

In another example, as illustrated in Fig. 10, suppose the probability of transitions $t_{1}, t_{2}, t_{3}$ being fired is $p_{1}, p_{2}$, and $p_{3}$, respectively. In $E P_{1}$ (see Fig. 10a), two events $e_{1}$ and $e_{2}$ are exposed to signal the state changes from $s_{0}$ to $s_{2}$ and $s_{3}$, respectively. In $E P_{2}$ (see Fig. 10b), three events $e_{0}^{\prime}, e_{1}^{\prime}$, and $e_{2}^{\prime}$ are exposed to signal the state changes from $s_{0}$ to $s_{1}, s_{1}$ to $s_{2}$, and $s_{1}$ to $s_{3}$, respectively. Intuitively, $E P_{1}$ reveals less transition information than $E P_{2}$ doe, because $E P_{1}$ abstracts the state changes from $s_{0}$ to $s_{2}, s_{3}$, and hides the intermediate state changes via $s_{1}$. This can be confirmed by calculating the information leakage of $E P_{1}$ and $E P_{2}$. According to the above discussion, we can derive that $I\left(G ; E P_{2}\right)=I\left(G ; E P_{1}\right)-p_{1} \log p_{1}$. Since $p_{1} \log p_{1} \leq 0$, we have $I\left(G ; E P_{1}\right) \leq I\left(G ; E P_{2}\right)$.

Event generation and propagation. Events for BPEL services can be generated by BPEL engines (e.g., Apache ODE [1]). For services implemented using other languages (e.g., Java, $\mathrm{C}$, or $\mathrm{C}++$ ), aspect-oriented programming techniques can be applied to generate events in a way transparent to service implementations. In addition, events can be propagated to service consumers using a pub/sub middleware [43] or using existing Web service protocols like WS-Eventing [69].

Parallel events. Besides the parallel events exposed simultaneously from different services (as discussed in Section 5), a single service may also involve concurrent executions (e.g., flows in BPEL) that generate interleaved events. The finite state machine model of the service can describe all the possible interleavings as different paths. Service providers can also choose to expose some of them (since different combinations correspond to the same concurrent execution paths) or all based on the testing requirements (e.g., examining every possible interleaving for race conditions [70]).

Composite Web services. A service may be composed of other services. The event interface of a composite service should be derived based on the event interfaces of its composed services. This can be done by aggregating events from the involved services into high-level events and constructing their causality relationships and conditions.

Test oracle. The test oracle issue is out of the scope of this paper. Service consumers can design a test oracle to verify testing results based on the specification of an SOA application. It is also possible to derive additional test oracles based on exposed events. This, however, may require semantic information linking the exposed events to the application logic. We plan to investigate this question in future work.

In practice, the oracle construction may be very costly. This may restrict the application of random and our hybrid test case generation. Some techniques, such as test case minimization [57], [58] and metamorphic testing [20], can be applied to alleviate this issue.

Trustability and granularity of event interface. It is possible that some service providers may publish an event interface that does not conform to its service implementation. This may cause the testing reports to become inaccurate. Similarly, some service providers may expose a finegrained event interface with more internal details, whereas another may expose a coarse-grained event interface with less internal details. The composition of event interfaces with different granularity may also cause the testing reports to become inaccurate.

One possible way to address this issue is to set up a feedback mechanism to evaluate the "testability" of a service, which can be seen as a kind of quality of the service. The "testability" of a service may include the availability of event interfaces for testing purposes, the quality and reputation of the event interfaces, and so on. For example, although a service may publish an inaccurate (or coarsegrained) event interface to obtain good test coverage reports (e.g., expose only a small subset of definition-use pairs), the service, however, is not well tested and may thus reveal many faults later on. Consequently, the service obtains a bad reputation and is assigned a poor testability score.

Nonfunctional testing. In this paper, we focused on how to make use of event exposure to increase the visibility of a service's functional testing for service consumers. Our approach can be adapted to support nonfunctional testing of services as well. For example, service providers can encapsulate information such as the execution time or resource usage into exposed events to help service consumers test a service's performance and diagnose 
performance bottlenecks. We plan to extend our approach to support nonfunctional testing of services in future work.

Handling side effects for service testing. To remove the side effects introduced in SOA testing, solutions have been proposed such as sandboxing services and invoking services with specific test modes during testing [18]. These solutions may have impact on test case generation and testing processes. For example, each involved service may provide a subset of data and resources to simulate the runtime situation for testing purposes. This data may suffice for testing the service itself, but may be inadequate to test a whole service composition. As a result, the generated test cases are incomplete and the service composition is not well covered during testing. In future work, we plan to investigate some techniques for service providers to customize the testing environment for service compositions based on their event interfaces.

In addition, for pay-per-use services without any sandbox, it is important to make testing effective. For example, to adequately cover a service composition, the constraintbased test case generation approach uses a smaller number of test cases (and thus has lower cost) than the hybrid and random solutions. In such cases, the testing cost should also be regarded as a factor in the testability of a service (i.e., the higher the cost to test a service, the lower the testability of the service). Service providers need to trade off the testability (and cost) against information leakage in test case generation.

\section{Related Work}

In this section, we review related work in the areas of service testing, test case generation, service interfaces, and runtime verification of services.

Service testing. Service testing has become an active area of research in the software engineering and has received much attention in recent years [4], [5], [7], [10], [17], [46], [55], [56], [80], [84]. Existing approaches can be classified into two main categories based on the roles involved in testing services: 1 ) from the perspective of service providers and 2) from the perspective of service consumers.

From the perspective of service providers, services need to be tested to conform to quality standards prior to release. Service providers usually have all the implementation details of their services (or partial details if third-party services are integrated to implement their services). Therefore, service providers can white-box test their services using control-flow and data-flow coverage strategies [44], [45], [55], [56], [81]. These approaches, however, are inadequate to white-box test a service composition involving third-party services because the implementations of third-party services are usually hidden.

Service consumers, on the other hand, need to know whether the selected third-party services work correctly when composed together to form new applications, even though each service has been tested individually by each service provider. Black-box testing approaches are often applied due to unavailable implementations of third-party services, including automatic test case generation for business protocols [42], testbeds to replace tested services [9], test case selection [57], and so on. These approaches can help to detect faults in a service composition. The limitation is that it is unclear to service consumers how adequately a service composition as a whole has been tested.

Whitening SOA testing. To gain confidence about how well a service composition has been tested, service consumers need to whiten SOA testing for service compositions. Li et al. [46] suggested that service providers design test cases based on their BPEL processes and provide the test cases to service consumers. The limitation is that service providers cannot anticipate all possible composition scenarios. Bartolini et al. [4], [5] proposed instrumenting each service with an intermediate service which provides coverage feedback for each third-party service to service consumers in unit testing. However, the coverage percentage provided by such an approach cannot be used to derive how adequately a whole service composition has been tested. Moreover, this approach does not address how to design test cases based on the feedback. We view this as a nontrivial step. Our approach addresses these two issues through the novel concept of event exposure from services that we developed. By observing events and matching them to feasible event sequences constructed from event interfaces, service consumers can determine the test coverage of a service composition as a whole. Test cases can also be designed effectively based on event interfaces, as we demonstrate. In addition, Rutherford et al. [65] developed a simulation approach to improve a test suite, whereas we make use of exposed events to improve test coverge for live service compositions.

Test case generation and selection. Many approaches have been proposed to generate test cases for Web services based on WSDL [3], [48], an ontology [71], a decision table [60], a graph [81], model checking [82], and a contract [25], to just name a few. Some of these approaches (e.g., contract-based solutions) are similar to our constraint-based approach, but they provided no solutions to trade off the effectiveness, efficiency, and information leakage in test case generation. In addition, to overcome the scalability issue, search-based techniques (e.g., genetic algorithms) can be used as an alternative to constraint solvers for constraint-based test case generation [54]. Differently, our hybrid test case generation allows service providers to customize the testing capacity by varying the tradeoff between the effectiveness, efficiency and information leakage, and thus complements existing approaches. It is possible to apply some searchbased techniques [54] instead of random testing in our hybrid solution to further improve the efficiency of test case generation. We plan to investigate this issue in future work.

Another important issue is test case selection. Mei et al. [57] proposed an approach to help service consumers prioritize test case selection for service regression testing based on the coverage of WSDL tags of the tested service. This approach is essentially a black-box testing approach. Li et al. [46] addressed the test case selection issue in service regression testing by collecting service execution traces in testing and compared it to the service implementation. Such an approach may not be feasible when the service implementation details are unavailable. Our event interfaces are designed mainly for whitening SOA applications, but the increased visibility based on event exposure can also be used to help select test cases in service regression testing. 
Testing component-based software. Many research efforts have been devoted to testing component-based software [13], [49], [50], wherein the source code and design of a component may also be unavailable. Therefore, metadata of components such as constraints on succeeding and preceding events are provided for testing purposes [14], [62]. This approach is similar to ours, in the sense that component vendors need to provide customers with interfaces and probes to observe internal states of components for testing purposes. The difference is that their approach applied constraint-based testing to examine the coverage of constraints inside each individual component, whereas our approach focuses on structural coverage of a service composition as a whole based on event exposure. As illustrated in Section 2, adequate testing of each service does not guarantee adequate testing of a service composition as a whole. Moreover, deciding what information component vendors have to provide is not specified in their approach. Instead, we offer approaches to derive and expose only necessary information for service consumers to adequately determine test coverage without releasing service implementation details. Beydeda and Gruhn [11] also proposed to provide the state machines of components and applied traditional structural testing techniques to test componentbased systems. This solution, however, reveals the implementation details of components. Differently, our solution applies event encapsulation techniques to expose only necessary information and protect service implementation details from being leaked.

Testing equivalence of processes. Much research has also been devoted to conformance testing of service specifications and their implementations [8], [27], [59], [67]. Our work also defines an equivalence relationship between the event interface and the service implementation. The difference is that existing work does conformance testing of service specification and implementation, whereas our approach determines the test coverage for white-box testing. Even though two processes are equivalent in terms of conformance testing, the test cases designed based on one process may not cover the same paths in the other process.

Service interface. Often, the implementation of a service is invisible to service consumers except for restricted service interfaces. Many researchers have studied how to enrich service interfaces to facilitate a service composition. Beyer et al. [12] proposed specifying constraints in service interfaces to define correctness requirements. Alfaro and Henzinger [26] proposed describing interfaces as automata to capture temporal aspects of constraints. Emmi et al. [30] proposed an approach based on assume-guarantee rules to check the compatibility of interface automata. Ye et al. [78], [79] proposed an atomicity-equivalent public view to check the atomicity property of a service composition. Industrial standards like the SCA Event Interface [63] and the WSEventing protocol [69] were proposed to expose and propagate events among services. However, all the aforementioned approaches do not address how to use service interfaces to white-box test a service composition. For example, an interface automaton [26] describes the temporal orders between the inputs and outputs. Such temporal orders are helpful to analyze the compatibility between interacting components, but they reveal little information about the test coverage inside a component (e.g., coverage of a definition-use pair). Our work contributes a new kind of service interface, namely, an event interface to reveal the test coverage information inside services and whiten the testing of services, and thus complements existing approaches.

Runtime verification of Web services. Besides service testing, service providers and consumers may also apply some runtime verification techniques to monitor and verify the properties of Web services, such as transactional requirements [34], data consistency [76], [75], conformance of interface contracts [29], [36], timing constraints [19], to just name a few. When some properties are violated at runtime, some mechanisms (e.g., compensation [79]) are usually needed to recover services from failures. Although runtime verification techniques can avoid the side effects introduced during service testing, recovering services from failures dynamically may cause unacceptable recovery costs [79]. In addition, runtime verification techniques can be applied to the scenarios where services evolve or are bound dynamically. Note that the concept of event exposure proposed in this paper is mainly for testing purposes, but the exposed events can also be used to support runtime verification of services.

\section{Conclusions}

Whitening the testing of service compositions is difficult because service providers usually reveal either no or only partial implementation details of their services to protect business interests. This paper presents a novel approach to whiten the testing of service compositions based on event exposure from Web services. By deriving event interfaces from service implementations, events are defined and exposed from services to accurately determine the test coverage of a service composition at runtime. In this way, service consumers can gain confidence on how adequately a service composition has been tested. To reduce information leakage, an approach is proposed to hide information from exposed events based on event encapsulation. The correctness of the solution is proven. In addition, a hybrid approach is proposed to design test cases based on event interfaces that can tradeoff effectiveness, efficiency, and information leakage. Algorithms to derive event interfaces are developed. The experimental results show that our approach outperforms existing approaches in terms of coverage percentage, fault-detection rate and effectiveness of test case generation.

\section{APPENDIX \\ Proof OF TheOREM 1}

The proof of Theorem 1 is to construct a one-to-one mapping between the structural elements inside a service composition and the exposed event sequences. For the DU pair, according to the definition of DU pair and the event interface for DU coverage criterion in Section 6, the mapping is straightforward. We only need to prove the correctness for the path coverage criterion. Given a path of a service composition, according to Algorithm 1, there exists 
a feasible event sequence for this path. Moreover, for two different paths of a service composition, the corresponding feasible event sequences are not identical because at least two different events will be exposed since the two paths differ by taking different branches from some locations. On the other hand, according to the event interface derived in Section 6.2.2, all the possible feasible event sequences are derived and exposed. Therefore, there is a one-to-one mapping between the paths inside a service composition and the exposed feasible event sequences.

\section{ACKNOWLEDGMENTS}

This research was supported in part by IBM's Center for Advanced Studies, an IBM Faculty Award, a Discover Accelerator Supplement and a Discovery grant from the Natural Sciences and Engineering Research Council of Canada, an Ontario Early Researcher Award, and a grant from the Mathematics of Information Technology and Complex Systems research network.

\section{REFERENCES}

[1] “ODE BPEL Engine," Apache, http://ode.apache.org, 2013.

[2] L. Ardissono, L. Console, A. Goy, G. Petrone, C. Picardi, M. Segnan, and D.T. Dupre, "Enhancing Web Services with Diagnostic Capabilities," Proc. Third European Conf. Web Services, p. 182, 2005.

[3] X. Bai, W. Dong, W. Tsai, and Y. Chen, "WSDL-Based Automatic Test Case Generation for Web Services Testing," Proc. IEEE Int'l Workshop Service-Oriented System Eng., pp. 207-212, 2005.

[4] C. Bartolini, A. Bertolino, S. Elbaum, and E. Marchetti, "Whitening SOA Testing," Proc. Seventh Joint Meeting of the European Software Eng. Conf. and the ACM SIGSOFT Symp. The Foundations of Software Eng., pp. 161-170, 2009.

[5] C. Bartolini, A. Bertolino, S. Elbaum, and E. Marchetti, "Bringing White-Box Testing to Service Oriented Architectures through a Service Oriented Approach," J. Systems and Software, vol. 84, pp. 655-668, Apr. 2011.

[6] C. Bartolini, A. Bertolino, F. Lonetti, and E. Marchetti, "Approaches to Functional, Structural and Security SOA Testing," Performance and Dependability in Service Computing: Concepts, Techniques and Research Directions, to be published, 2011

[7] C. Bartolini, A. Bertolino, E. Marchetti, and A. Polini, "Towards Automated WSDL-Based Testing of Web Services," Proc. Sixth Int'l Conf. Service-Oriented Computing, pp. 524-529, 2008.

[8] L. Bentakouk, P. Poizat, and F. Zaïdi, "A Formal Framework for Service Orchestration Testing Based on Symbolic Transition Systems," Proc. 21st IFIP WG 6.1 Int'l Conf. Testing of Software and Comm. Systems and Ninth Int'l FATES Workshop, pp. 16-32, 2009

[9] A. Bertolino, G. Angelis, L. Frantzen, and A. Polini, "Model-Based Generation of Testbeds for Web Services," Proc. 20th IFIP TC 6/ WG 6.1 Int'l Conf. Testing of Software and Communicating Systems: Eighth Int'l Workshop, pp. 266-282, 2008.

[10] A. Bertolino and A. Polini, "SOA Test Governance: Enabling Service Integration Testing Across Organization and Technology Borders," Proc. IEEE Int'l Conf. Software Testing, Verification, and Validation Workshops, pp. 277-286, 2009.

[11] S. Beydeda and V. Gruhn, "An Integrated Testing Technique for Component-Based Software," Proc. ACS/IEEE Int'l Conf. Computer Systems and Applications, p. 328, 2001.

[12] D. Beyer, A. Chakrabarti, and T.A. Henzinger, "Web Service Interfaces," Proc. Int'l Conf. World Wide Web, pp. 148-159, 2005

[13] R.V. Binder, Testing Object-Oriented Systems: Models, Patterns, and Tools. Addison-Wesley Longman Publishing Co., Inc., 1999.

[14] L.C. Briand, Y. Labiche, and M.M. Sówka, "Automated, ContractBased User Testing of Commercial-Off-the-Shelf Components," Proc. 28th Int'l Conf. Software Eng., pp. 92-101, 2006.
[15] L.C. Briand, Y. Labiche, and Y. Wang, "A Comprehensive and Systematic Methodology for Client-Server Class Integration Testing," Proc. 14th Int'l Symp. Software Reliability Eng., p. 14, 2003.

[16] A. Bucchiarone, H. Melgratti, and F. Severoni, "Testing Service Composition," Proc. Eighth Argentine Symp. Software Eng., 2007.

[17] G. Canfora and M. Di Penta, "Testing Services and Service-Centric Systems: Challenges and Opportunities," IT Professional, vol. 8 , no. 2, pp. 10-17, Mar./Apr. 2006

[18] G. Canfora and M. Di Penta, "Service-Oriented Architectures Testing: A Survey," Software Eng., pp. 78-105, 2009.

[19] T.-D. Cao, T.-T. Phan-Quang, P. Felix, and R. Castanet, "Automated Runtime Verification for Web Services," Proc. IEEE Int'l Conf. Web Services, pp. 76-82, 2010.

[20] W. Chan, S. Cheung, and K. Leung, "A Metamorphic Testing Approach for Online Testing of Service-Oriented Software Applications," Int'l J. Web Services Research, vol. 4, no. 2, pp. 6181, 2007.

[21] K. Chandy, "Event-Driven Applications: Costs, Benefits and Design Approaches," Proc. Gartner Application Integration and Web Services Summit Conf., 2006.

[22] K. Chatzikokolakis, T. Chothia, and A. Guha, "Statistical Measurement of Information Leakage," Proc. Tools and Algorithms for the Construction and Analysis of Systems Conf., pp. 390-404, 2010.

[23] L.A. Clarke, "A System to Generate Test Data and Symbolically Execute Programs," IEEE Trans. Software Eng., vol. 2, no. 3, pp. 215-222, Sept. 1976.

[24] J.C. Corbett, M.B. Dwyer, J. Hatcliff, S. Laubach, C.S. Păsăreanu, and H. Zheng, "Bandera: Extracting Finite-State Models from Java Source Code," Proc. Int'l Conf. Software Eng., pp. 439-448, 2000.

[25] G. Dai, X. Bai, Y. Wang, and F. Dai, "Contract-Based Testing for Web Services," Proc. 31st Ann. Int'l Computer Software and Applications Conf., vol. 1, pp. 517-526, 2007.

[26] L. de Alfaro and T.A. Henzinger, "Automata," Proc. Eighth European Software Eng. Conf., pp. 109-120, 2001.

[27] R. De Nicola and M. Hennessy, "Testing Equivalence for Processes," Proc. Int'l Colloquium Automata Languages and Programming, pp. 548-560, 1983.

[28] R.A. DeMillo, R.J. Lipton, and F.G. Sayward, "Hints on Test Data Selection: Help for the Practicing Programmer," Computer, vol. 11, pp. 34-41, Apr. 1978

[29] D. Dranidis, E. Ramollari, and D. Kourtesis, "Run-Time Verification of Behavioural Conformance for Conversational Web Services," Proc. IEEE Seventh European Conf. Web Services, pp. 139-147, 2009

[30] M. Emmi, D. Giannakopoulou, and C.S. Păsăreanu, "AssumeGuarantee Verification for Interface Automata," Proc. 15th Int'l Symp. Formal Methods, pp. 116-131, 2008.

[31] I. Epifani, C. Ghezzi, and G. Tamburrelli, "Change-Point Detection for Black-Box Services," Proc. 18th ACM SIGSOFT Int'l Symp. Foundations of Software Eng. pp. 227-236, 2010.

[32] H. Foster, S. Uchitel, J. Magee, and J. Kramer, "Model-Based Verification of Web Service Compositions," Proc. 18th IEEE Int'l Conf. Automated Software Eng., pp. 152-161, 2003.

[33] X. Fu, T. Bultan, and J. Su, "Analysis of Interacting Bpel Web Services," Proc. 13th Int'l Conf. World Wide Web, pp. 621-630, 2004.

[34] W. Gaaloul, S. Bhiri, and M. Rouached, "Event-Based Design and Runtime Verification of Composite Service Transactional Behavior," IEEE Trans. Services Computing, vol. 3, no. 1, pp. 32-45, Jan.-Mar. 2010.

[35] V. Ganesh and D.L. Dill, "A Decision Procedure for Bit-Vectors and Arrays," Proc. 19th Int'l Conf. Computer Aided Verification, pp. 519-531, 2007

[36] S. Hallé, T. Bultan, G. Hughes, M. Alkhalaf, and R. Villemaire, "Runtime Verification of Web Service Interface Contracts," Computer, vol. 43, no. 3, pp. 59-66, Mar. 2010.

[37] J.E. Hanson, P. Nandi, and S. Kumaran, "Conversation Support for Business Process Integration," Proc. Sixth Int'l Enterprise Distributed Object Computing Conf., pp. 65-74, 2002.

[38] J. Hodges Jr and E. Lehmann, "Estimates of Location Based on Rank Tests," The Annals of Math. Statistics, vol. 34, pp. 598-611 1963.

[39] M. Hutchins, H. Foster, T. Goradia, and T. Ostrand, "Experiments of the Effectiveness of Dataflow- and Controlflow-Based Test Adequacy Criteria," Proc. 16th Int'l Conf. Software Eng., pp. 191200, 1994.

[40] "Loan Approval," IBM, http://www.ibm.com/developerworks / webservices/library/ws-bpelcol5/, 2013. 
[41] Z. Jin and A.J. Offutt, "Coupling-Based Criteria for Integration Testing," The J. Software Testing, Verification, and Reliability, vol. 8, pp. 133-154, 1998.

[42] K. Kaschner and N. Lohmann, "Automatic Test Case Generation for Interacting Services," Proc. Int'l Conf. Service-Oriented Computing Workshops, pp. 66-78, 2008.

[43] G. Li, V. Muthusamy, and H.-A. Jacobsen, "A Distributed ServiceOriented Architecture for Business Process Execution," ACM Trans. Web, vol. 4, no. 1, pp. 1-33, 2010.

[44] L. Li, W. Chou, and W. Guo, "Control Flow Analysis and Coverage Driven Testing for Web Services," Proc. IEEE Int'l Conf. Web Services, pp. 473-480, 2008.

[45] Z. Li, W. Sun, Z.B. Jiang, and X. Zhang, "BPEL4WS Unit Testing: Framework and Implementation," Proc. IEEE Int'l Conf. Web Services, pp. 103-110, 2005.

[46] Z.J. Li, H.F. Tan, H.H. Liu, J. Zhu, and N.M. Mitsumori, "BusinessProcess-Driven Gray-Box SOA Testing," IBM Systems J., vol. 47, no. 3, pp. 457-472, 2008.

[47] D.C. Luckham, The Power of Events: An Introduction to Complex Event Processing in Distributed Enterprise Systems. Addison-Wesley, 2001.

[48] C. Ma, C. Du, T. Zhang, F. Hu, and X. Cai, "WSDL-Based Automated Test Data Generation for Web Service," Proc. Int'l Conf. Computer Science and Software Eng., vol. 2, pp. 731-737, 2008.

[49] L. Mariani, "Behavior Capture and Test for Verifying Evolving Component-Based Systems," Proc. Int'l Conf. Software Eng., pp. 7880, 2004.

[50] L. Mariani, S. Papagiannakis, and M. Pezze, "Compatibility and Regression Testing of Cots-Component-Based Software," Proc. 29th Int'l Conf. Software Eng., pp. 85-95, 2007.

[51] L. Mather and E. Oswald, "Pinpointing Side-Channel Information Leaks in Web Applications," J. Cryptographic Eng., vol. 2, pp. 131177, 2012.

[52] J. Mayer and C. Schneckenburger, "An Empirical Analysis and Comparison of Random Testing Techniques," Proc. ACM/IEEE Int'l Symp. Empirical Software Eng., pp. 105-114, 2006.

[53] T. McCabe, "A Complexity Measure," IEEE Trans. Software Eng., vol. 2, no. 4, pp. 308-320, Dec. 1976.

[54] P. McMinn, "Search-Based Software Test Data Generation: A Survey: Research Articles," Software Testing, Verification, and Reliability, vol. 14, no. 2, pp. 105-156, June 2004.

[55] L. Mei, W. Chan, and T. Tse, "Data Flow Testing of ServiceOriented Workflow Applications," Proc. 30th Int'l Conf. Software Eng., pp. 371-380, 2008.

[56] L. Mei, W.K. Chan, and T.H. Tse, "Data Flow Testing of Service Choreography," Proc. Seventh Joint Meeting of the European Software Eng. Conf. and the ACM SIGSOFT Symp. The Foundations of Software Eng., pp. 151-160, 2009.

[57] L. Mei, W.K. Chan, T.H. Tse, and R.G. Merkel, "Tag-Based Techniques for Black-Box Test Case Prioritization for Service Testing," Proc. Ninth Int'l Conf. Quality Software, pp. 21-30, 2009.

[58] L. Mei, Z. Zhang, W.K. Chan, and T.H. Tse, "Test Case Prioritization for Regression Testing of Service-Oriented Business Applications," Proc. 18th Int'l Conf. World Wide Web, pp. 901-910, 2009.

[59] H. Muccini, A. Bertolino, and P. Inverardi, "Using Software Architecture for Code Testing," IEEE Trans. Software Eng., vol. 30, no. 3, pp. 160-171, Mar. 2004.

[60] S. Noikajana and T. Suwannasart, "Web Service Test Case Generation Based on Decision Table (Short Paper)," Proc. Eighth Int'l Conf. Quality Software, pp. 321-326, 2008.

[61] "Book Ordering," Oracle, http://www.oracle.com/technology/ sample_code/products/bpel/index.html, 2013.

[62] A. Orso, M.J. Harrold, and D.S. Rosenblum, "Component Metadata for Software Engineering Tasks," Proc. Revised Papers from the Second Int'l Workshop Eng. Distributed Objects, pp. 129-144, 2001.

[63] “SCA Event Processing," OSOA, http://www.osoa.org/, 2013.

[64] C. Peltz, "Web Services Orchestration and Choreography," Computer, vol. 36, no. 10, pp. 46-52, Oct. 2003.

[65] M.J. Rutherford, A. Carzaniga, and A.L. Wolf, "Evaluating Test Suites and Adequacy Criteria Using Simulation-Based Models of Distributed Systems," IEEE Trans. Software Eng., vol. 34, no. 4, pp. 452-470, July 2008.

[66] C.E. Shannon, "A Mathematical Theory of Communication," ACM SIGMOBILE Mobile Computing and Comm. Rev., vol. 5, no. 1, pp. 355, Jan. 2001.
[67] J. Tretmans, "Test Generation with Inputs, Outputs, and Quiescence," Proc. Second Int'l Workshop Tools and Algorithms for Construction and Analysis of Systems, pp. 127-146, 1996.

[68] "Web Services Description Language," W3C, http://www.w3. org/TR/wsdl, 2013.

[69] “Ws-Eventing, Owl-s," W3C, http://www.w3.org, 2013.

[70] L. Wang and S.D. Stoller, "Runtime Analysis of Atomicity for Multi-Threaded Programs," IEEE Trans. Software Eng., vol. 32, no. 2, pp. 93-110, Feb. 2006

[71] Y. Wang, X. Bai, J. Li, and R. Huang, "Ontology-Based Test Case Generation for Testing Web Services," Proc. Eighth Int'l Symp. Autonomous Decentralized Systems, pp. 43-50, 2007.

[72] E.J. Weyuker, "Axiomatizing Software Test Data Adequacy," IEEE Trans. Software Eng., vol. 12, no. 12, pp. 1128-1138, Dec. 1986.

[73] F. Wilcoxon, "Individual Comparisons by Ranking Methods," Biometrics Bull., vol. 1, no. 6, pp. 80-83, 1945.

[74] "Supply Chain Management," WS-I., http://www.ws-i.org/ deliverables/workinggroup.aspx?wg=sampleapps, 2013.

[75] G. Wu, J. Wei, C. Ye, X. Shao, H. Zhong, and T. Huang, "Runtime Monitoring of Data-Centric Temporal Properties for Web Services," Proc. IEEE Int'l Conf. Web Services, pp. 161-170, 2011.

[76] G. Wu, J. Wei, C. Ye, H. Zhong, and T. Huang, "Detecting Data Inconsistency Failure of Composite Web Services through Parametric Stateful Aspect," Proc. IEEE Int'l Conf. Web Services, pp. 6875, 2010.

[77] W. Xu, J. Offutt, and J. Luo, "Testing Web Services by XML Perturbation," Proc. IEEE 16th Int'l Symp. Software Reliability Eng., pp. 257-266, 2005.

[78] C. Ye, S.C. Cheung, and W.K. Chan, "Publishing and Composition of Atomicity-Equivalent Services for B2B Collaboration," Proc. 28th Int'l Conf. Software Eng., pp. 351-360, 2006.

[79] C. Ye, S.C. Cheung, W.K. Chan, and C. Xu, "Atomicity Analysis of Service Composition across Organizations," IEEE Trans. Software Eng., vol. 35, no. 1, pp. 2-28, Jan./Feb. 2009.

[80] C. Ye and H.-A. Jacobsen, "White-Box Testing of Service Compositions via Event Interfaces," technical report, Univ. of Toronto, http://msrg.org/papers/Ye10b. 2010.

[81] Y. Yuan, Z. Li, and W. Sun, "A Graph-Search Based Approach to BPEL4Ws Test Generation," Proc. Int'l Conf. Software Eng. Advances, p. 14, 2006.

[82] Y. Zheng, J. Zhou, and P. Krause, "An Automatic Test Case Generation Framework for Web Services," J. Software, vol. 2, no. 3, pp. 64-77, 2007.

[83] H. Zhu, P.A.V. Hall, and J.H.R. May, "Software Unit Test Coverage and Adequacy," ACM Computing Surveys, vol. 29, no. 4, pp. 366-427, 1997

[84] H. Zhu and Y. Zhang, "Collaborative Testing of Web Services," IEEE Trans. Services Computing, vol. 5, no. 1, pp. 116-130, Jan.-Mar. 2012.

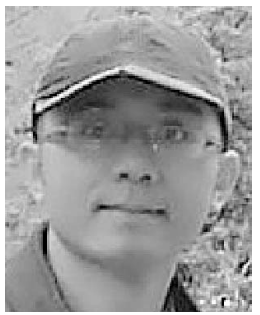

Chunyang Ye received the BEng degree from the University of Science and Technology of China in 2000, the MEng degree from the Chinese Academy of Sciences in 2003, and the PhD degree from the Hong Kong University of Science and Technology in 2008. He visited the University of Toronto as a postdoctoral fellow in the Middleware Systems Research Group from 2009 to 2011. His research interests include software engineering issues on business process management, distributed and pervasive middleware systems, services, and cloud computing. He is a member of the Application and Middleware Systems Research Group at the Technical University of Munich, and an associate research professor in the Technology Center of Software Engineering, Institute of Software, Chinese Academy of Sciences.

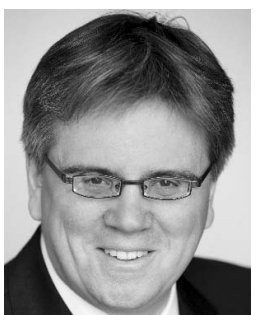

Hans-Arno Jacobsen is a professor of computer engineering and computer science and directs the Middleware Systems Research Group at the University of Toronto. His principal areas of research include the design and the development of middleware systems and distributed systems. His research revolves around publish/subscribe, content-based routing, event processing, and aspect-orientation. $\mathrm{He}$ is a senior member of the IEEE. 